\title{
Im Spannungsfeld zwischen hochdeutscher Norm und niederdeutscher Sprachkompetenz
}

Codemixing in einem niederdeutschen Brief des beginnenden 17. Jahrhunderts

\author{
Nicolaus Janos Raag | ORCID: 0000-0002-8234-0142 \\ Institutionen för moderna språk, Uppsala universitet, Uppsala, Schweden \\ nicolaus.raag@moderna.uu.se
}

\begin{abstract}
This article contains an edition of a 17th-century Low German letter addressed to the German congregation in Stockholm. Seen against the shift of writing language from Low to High German, this letter is analyzed in respect to code-mixing, which is shown to fulfill a communicative function. Furthermore the author here suggests that the code-mixing observed in the letter can be described as congruent lexicalization, where Low German syntactic structures are filled with both Low and High German lexical material.
\end{abstract}

\section{Keywords}

historical contact linguistics - Middle Low German - Early New High German - early modern correspondence - code-mixing - Stockholm

\section{Einleitung}

Vor dem Hintergrund des Schreibsprachenwechsels vom Nieder- zum Hochdeutschen lassen sich im frühneuzeitlichen Archivmaterial der Deutschen St. Gertruds Gemeinde zu Stockholm einige interessante Beobachtungen machen, die ein Licht auf denSprachgebrauch dieserZeit werfen. MitStockholm nehmen wir gewissermaßen die Peripherie des deutschen Sprachraums in den Blick, also ein Sprachmilieu, in dem Deutsch eine (wenn auch große und bedeutende) Minderheitensprache war. Hier war das Niederdeutsche seit dem 
Spätmittelalter eine lebendige geschriebene und gesprochene Sprache und der Anteil der deutschsprachigen Bevölkerung war erheblich (Weinauge, 1942: 70; Ahnlund, 1953: 300; Lager, 1962: 122 f.; Dahlbäck, 1988: 52; Sidén, 2008: 3). Ein Blick in die frühneuzeitlichen Rechnungsakten der Stockholmer deutschen Gemeinde zeigt, dass das Niederdeutsche neben dem Hochdeutschen auch am Übergang vom 16. zum 17. Jahrhundert noch Anwendung in der internen Kommunikation der Gemeinde fand, beispielsweise in der Buchführung oder in kürzeren, die Geldgeschäfte betreffenden Schreiben. Dementsprechend darf man davon ausgehen, dass das Niederdeutsche im Stockholm dieser Zeit auch noch eine gesprochene Sprache war.

Gleichzeitig verwendete die Gemeinde in ihrer Außenkommunikation aber schon das Hochdeutsche, wie etwa die Briefe an den Ältestenrat der deutschen Gemeinde zeigen, die schon in den ersten Jahrzehnten ihres Bestehens (bis 1650) fast ausschließlich Hochdeutsch sind - sieht man von einigen lateinischen und schwedischen Ausnahmen ab. ${ }^{1}$ In diesem Kontext fallen dann zwei der frühen Schreiben an den Ältestenrat besonders auf, namentlich der in niederdeutsch-hochdeutscher Mischsprache verfasste Brief eines Hannß Bartteram vom 28. Juli 16o1 sowie ein einzelner (fast) vollständig niederdeutscher Brief eines Mattewes Schroder vom 7. März 16o2. Dieser Brief weist nun einige vor dem o. g. Hintergrund des Schreibsprachenwechsels und der sprachlichen Verhältnisse in Stockholm beachtenswerte Phänomene des Codemixings von Nieder- und Hochdeutsch auf und soll in dem hier vorliegenden ersten Artikel editiert und unter diesem Aspekt näher betrachtet werden. In einem zweiten Artikel (in Vorbereitung) soll dann das Codemixing in Hannß Bartterams Brief betrachtet werden. So soll auch dazu beigetragen werden, eine auffallende Lücke in der Erforschung des Übergangs vom Niederdeutschen zum Hochdeutschen in Schweden zu füllen.

Seit Anfang der 1990er Jahre sind Mehrsprachigkeit und Sprachmischung mehr und mehr in den Fokus der historischen Sprachforschung gerückt und historische, gemischtsprachige Texte wurden als früheste Beispiele von schriftlichem Codemixing betrachtet, die auch Aufschluss über den Sprachgebrauch historischer Sprachbenutzer geben können. Dieses zunehmende Interesse fand in jüngerer Zeit seinen Niederschlag in einer ganzen Reihe von einschlägigen Sammelbänden, so zum Beispiel Schendl und Wright (2011b), Wich-Reif (2016), Pahta, Skaffari und Wright (2018), Glaser, Prinz und Ptashnyk (i. E.). In Fortsetzung dieser Tradition soll hier auch der Frage nach der Funktion des Codemixings in der historischen Kommunikationssituation

1 Bei diesen Ausnahmen handelt es sich meist um Schreiben schwedischer (kirchlicher) Institutionen, wie etwa den lateinischen Schreiben des Erzbischofs in Uppsala oder den schwedischen Schreiben des Stockholmer Konsistoriums. 
nachgegangen werden. Es wird davon ausgegangen, dass Codemixing kommunikative Bedeutung vermittelt, was sich auch anhand von Mattewes Schroders Brief zeigen lässt (s. Abschnitt 4.1.2, 4.3). Als ein wichtiger sprachhistorischer Kontext für die Kommunikationssituation, in der Mattewes' Brief geschrieben wird, ist der erwähnte Schreibsprachenwechsel vom Nieder- zum Hochdeutschen zu beachten (s. Abschnitt 2.1). So soll auch ein Beitrag zur Klärung der Frage geleistet werden, was dieser Schreibsprachenwechsel nicht nur für professionelle Schreiber, sondern auch für die breite Masse - hier am Beispiel eines Handwerkers - bedeutete.

Für die formale Analyse der einzelnen Codemixing-Phänomene soll hierfür auf das von Muysken (2000) vorgeschlagene Modell zurückgegriffen werden (s. Abschnitt 4.1.1), wobei von der Hypothese ausgegangen wird, dass das Codemixing hier durch die nahe Verwandtschaft und damit verbunden die strukturelle Nähe der beiden Kontaktsprachen Niederdeutsch-Hochdeutsch bedingt und begünstigt wird. Bevor aber in Abschnitt 4 der theoretische Hintergrund besprochen und diese Analysen vorgelegt werden sollen und in Abschnitt 5 noch eine Zusammenfassung unter Rückgriff auf o. g. Hypothesen und ein Ausblick auf weitere Studien gegeben werden soll, wird zunächst näher auf den historischen Hintergrund einzugehen sein (Abschnitt 2) und es wird Mattewes Schroders Brief editiert und in seinem Inhalt und seiner äußeren Form besprochen (Abschnitt 3).

\section{Historischer Hintergrund}

\subsection{Schreibsprachenwechsel}

Wie erwähnt stellt der Schreibsprachenwechsel einen wichtigen sprachhistorischen Kontext dar, vor dem das Codemixing im vorliegenden Brief zu verstehen ist. In der Literatur wurde verschiedentlich darauf hingewiesen, dass Codemixing-Phänomene insbesondere auch vor dem Hintergrund eines (Schreib-)Sprachwechsels zu beobachten sind, z. B. wird dies von Lüdi (1985: 165-168) für die Ratsprotokolle in Freiburg im Üechtland (Schweiz) vom Beginn des 15. Jahrhunderts nach einem Übergang von einer lokalen Schreibvarietät des Französischen zum Deutschen beobachtet oder von Schendl und Wright (2011a: 21 f.) für das Englische in halb-offiziellen Briefen des 15. Jahrhunderts, das in dieser Domäne das Französische und Lateinische erst seit dem Ende des 14. Jahrhunderts nach und nach abzulösen begonnen hatte. Ein solcher Zusammenhang lässt sich auch im vorliegenden Material nachvollziehen. ${ }^{2}$

2 Zur Rolle von Sprachmischung beim Sprachwechsel einer Sprechergemeinschaft und dem damit unter Umständen verbundenen Absterben ihrer ursprünglichen Sprache, vgl. auch 
Im Verlauf des 16. und 17. Jahrhunderts wird das Niederdeutsche als Schreibsprache in Norddeutschland und im weiteren Ost- und Nordseeraum vom Hochdeutschen abgelöst (vgl. u. v. a. Gabrielsson, 1983; Sodmann, 1983, 2000; Polenz, 2000: 258-269). Dieser Vorgang vollzieht sich nicht abrupt, vielmehr lassen sich laut Gabrielsson (1983: 126-130) drei Phasen unterscheiden, die schematisch eine gewisse Reihenfolge abbilden, sich in Wirklichkeit aber als nicht klar voneinander abgegrenzt beobachten lassen. In der ersten Phase bleibt die zugrundeliegende sprachliche Struktur niederdeutsch, in die dann vereinzelte hochdeutsche Elemente eingefügt werden (Gabrielsson, 1983: 127 spricht von „Eindringlingen“). Neben hochfrequenten Funktionswörtern sind dies zum Beispiel auch Titel, Anreden oder einige formelhafte Wendungen. In dieser Phase lässt sich auch der hier zu editierende und analysierende Brief des Mattewes Schroder verorten.

Die zweite Phase ist die eigentliche Übergangszeit. Sie ist durch die Bemühungen der Sprachträger gekennzeichnet, niederdeutsche Sprachformen durch bekannte Gleichungen zu verhochdeutschen. In dieser Phase begegnet auch eine niederdeutsch-hochdeutsche Mischsprache (Gabrielsson, 1983: 128). In der dritten Phase ist die zugrundeliegende sprachliche Struktur dann hochdeutsch, es begegnen aber noch viele niederdeutsche Einschübe und Sprachreste (Gabrielsson, 1983: 129).

Gegenüber der sehr breiten Literatur zum Schreibsprachenwechsel vom Nieder- zum Hochdeutschen in Norddeutschland gibt es zu Skandinavien relativ wenige Untersuchungen ${ }^{3}$. Laut Mähl (2012: 630) war Deutsch in Skandinavien bis Mitte des 16. Jahrhunderts weitestgehend Niederdeutsch. Am frühesten vollzog sich der Schreibsprachenübergang in Kopenhagen, wo der dänischnorwegische König Hans seit 1508 neben der dänischen auch eine deutsche Kanzlei unterhielt (vgl. Christensen, 1993: 55). Bis 1550 wird dort die niederdeutsche Kanzleisprache durch eine hochdeutsche ersetzt (Winge, 1987: 75).

Nach der Auflösung der Kalmarer Union richtete der schwedische Regent Gustav Wasa in Stockholm eine Kanzlei ein, in der auch deutsche Schreiber und Sekretäre tätig waren, die für die Korrespondenz mit dem Ausland, insbesondere mit deutschen Fürsten und Städten, benötigt wurden (Edén, 1899: 11, 36-40, 54-57; Upmark, 1912: 15; Svalenius, 1991: 82-87). Auch wenn die meisten der deutschen Schreiben dem Schlossbrand vom 7. Mai 1697 zum

Myers-Scotton (1993: 223-226) und Muysken (2000: 263-265), eher kritisch dazu z. B. Clyne (2003: 190 f.).

3 Dies zeigt auch ein Blick in die umfangreiche Bibliographie zum Schreibsprachenwechsel von Peters und Nagel (2018) in der über die zahlreichen Publikationen von Birgit Christensen und Vibeke Winge zu Dänemark hinaus nur wenige Beiträge zum Schreibsprachenwechsel in Skandinavien aufgeführt sind. Auch Mähl (2012: 631) nennt dies als ein Forschungsdesiderat. 
Opfer gefallen sind, lassen sich diese anhand der Titularregistratur ab $15^{22}$ nachweisen (Svalenius, 1991: 82 f.).

Auch in der Kanzlei Gustav Wasas setzt sich laut Mähl (2012: 630 f.) das Hochdeutsche als Schreibsprache durch und nach 1530 wird das Niederdeutsche in schwedischen Urkunden und Briefen nur noch selten gebraucht (vgl. Gabrielsson, 1983: 131). Allerdings fehlen tiefergehende Untersuchungen zu Stockholm. Für den östlichen Reichsteil des damaligen Schwedens, das heutige Finnland, hat Katara (1936: 182-184; 1950) darauf hingewiesen, dass das Niederdeutsche, das im 14. und 15. Jahrhundert Sprache des finnischen Außenhandels war, im 16. Jahrhundert adressatenabhängig vom Hochdeutschen bzw. Schwedischen ersetzt wurde. In den Quittungen von Kaufleuten und Handwerkern blieb es jedoch bis ins 17. Jahrhundert in Gebrauch (Katara und Kuujo, 1958: 6 f.; Katara, 1950).

\subsection{Kurze Geschichte der Deutschen St. Gertruds Gemeinde bis zur Wende zum 17.Jahrhundert}

Nachdem Gustav Wasa am 6. Juni 1523 zum König gewählt worden war, wurde in Schweden die lutherische Reformation eingeführt. Die neue Glaubensausrichtung weckte die Frage nach muttersprachlichem Gottesdienst auch für Deutsche und Finnen, die nach den Schweden die beiden größten Bevölkerungsgruppen in Stockholm stellten. Einer der frühen Vertrauten Gustav Wasas war der in der deutschen Kanzlei des Königs tätige Nicolaus Stecker (auch Stecher), der wie Martin Luther in Eisleben geboren war. Im Sommer 1524 wurde er zum ersten evangelischen Gemeindepfarrer in der Stadtkirche St. Nikolai ernannt (Carlsson, 1922: 8o f.), wo er - der erst kurz zuvor nach Schweden gekommen war - auf Deutsch gepredigt haben dürfte. Allerdings verließ er Schweden schon nach wenigen Jahren wieder, im Mai 1527 wird er zum letzten Mal als Pfarrer erwähnt (Carlsson, 1922: 88). Seine Nachfolger waren Schweden und es ist unbekannt, ob auch nach Steckers Abreise noch deutschsprachige Gottesdienste in der Stadtkirche St. Nikolai abgehalten werden konnten, etwa durch einen deutschen Kaplan oder Prediger. Sollte dies der Fall gewesen sein, so fand diese Praxis jedenfalls 1536 ein abruptes Ende, als die sogenannte Pulverkonspiration aufgedeckt wurde. Es handelte sich hierbei um ein versuchtes Attentat auf König Gustav, hinter dem sieben der deutschen Kaufleute der Stadt gemeinsam mit dem schwedischen Münzmeister standen, die den König als Hindernis ihrer Interessen sahen (Schieche, 1952: 29). Einer der Verschwörer, Clement Renzel (auch Rensel), war „seit 1533 Kirchenvorsteher bei St. Nikolai“ und ein weiterer, Hans Bökman, hatte bis 1529 nicht nur das gleiche Amt begleitet, sondern sich auch als eifriger Verfechter des evangelischen Glaubens hervorgetan (Schieche, 1952: 26, 29). Nachdem der Attentatsversuch ans Licht gekommen war, wurden die 
Deutschen wohl aus der Stadtkirche verdrängt (Ahnlund, 1929: 24) und sahen sich also gezwungen, sich andernorts in der Stadt nach einem geeigneten Raum für ihren Gottesdienst umzutun.

Aus einer Bittschrift vom März 1594 von „SamPtliche Deutzsche gemeine Burgerschafft vnnd frembde zu Stockholm" an König Sigismund geht hervor, dass die deutschen Gottesdienste in einer Klosterkirche abgehalten wurden. ${ }^{4}$ Bei dieser Klosterkirche dürfte es sich um die Kirche des aufgelassenen Dominikanerklosters handeln, die seit dem Herbst des Jahres 1533 für finnischsprachige Gottesdienste genutzt worden war. Sowohl Finnen als auch Deutsche nutzten die Klosterkirche für ihre Gottesdienste bis zu deren Abriss im Jahr 1547. Danach und bis zur offiziellen Anerkennung der Gemeinde nutzten Deutsche wie Finnen andere Räumlichkeiten für ihre Gottesdienste (Kjöllerström, 1935: 38), möglicherweise die Kapellen in den beiden außerhalb der eigentlichen Stadt gelegenen Siedlungen Norrmalm und Södermalm oder Privathäuser. Wer in dieser Zeit die deutschsprachigen Gottesdienste abgehalten hat, ist heute unbekannt, aber in den Jahren 1556 und 1557 soll ein deutscher Prediger namens Johannes Stägemann ein Gehalt aus der königlichen Rentenkammer bezogen haben, weshalb es auch nicht völlig undenkbar ist, dass die Deutschen nun wieder die Stadtkirche für ihre Gottesdienste nutzen durften (Kjöllerström, 1935: 36, Fußnote 46; Schieche, 1952: 38). Mit dem Prediger Stägemann (in den Akten Joen Stegemann, Stäkemann) beginnt eine im Prinzip ungebrochene series pastorum für die deutsche Gemeinde in Stockholm (vgl. Hellström, 1951: 556 ff.).

Die deutschen Bürger der Stadt waren unzufrieden, dass ihnen ein passender Raum für ihren Gottesdienst fehlte und beklagten sich bei König Johann III. (Murray, 1949: 95). Dieser erteilte ihnen am 20. Juli 1570 die Erlaubnis, den Konventssaal des ehemaligen Franziskanerklosters auf Gråmunkeholm (heute Riddarholm) zu nutzen und ausbauen zu lassen, bis dass sie einen besseren Raum erhalten konnten (Rothlieb, 1822: 45). Nicht einmal ein Jahr später, am 8. März 1571, fertigte der König einen Privilegienbrief aus, der es den Deutschen erlaubte, eine Kirche an dem Ort zu errichten, wo sich früher das Dominikanerkloster befunden hatte und „sielffwe förschaffe sigh lärde och gudfruchtige predicanter" [sich selbst gelehrte und gottesfürchtige Prediger zu verschaffen] (SE/SSA/oo17/J I b/2; abgedruckt in Schieche, 1952: 154-155). Der Privilegienbrief kann als eine indirekte Anerkennung der deutschen Gemeinde als eigenständige Institution gesehen werden. Der Kirchenbau kam möglicherweise aus ökonomischen Gründen nie zustande. Stattdessen wurde den Deutschen gemeinsam mit den Finnen das von der Krone konfiszierte

4 Eine Abschrift bzw. Konzept der Bittschrift findet sich in St. Gertruds Kirchenarchiv SE/ SSA/oo17/J II/1/37-41; ein Abdruck dieses Konzepts bei Lüdeke (1823: 68-72). 
Gildehaus St. Gertrud zur Nutzung für Gottesdienste überlassen. Die Finnen nutzten dieses Gebäude schon seit 1561 (Lüdeke, 1791: 8) und es wurde für lange Zeit ,Finnische Kirche' genannt, auch dann noch, als dort ab 1576 die deutschen Gottesdienste gehalten wurden. Damit das Gildehaus seinen neuen Zweck erfüllen konnte, wurde es zwischen 1576 und 1594 zu einer einschiffigen Kapelle umgebaut, indem es nach Westen hin verlängert und „mit Dach, Giebel, Fenstern, Stühlen und Gewölben“ versehen wurde (Lüdeke, 1823: 186). Die Kosten für den Umbau wurden von der deutschen Gemeinde bestritten, die über ganz andere Möglichkeiten und Verbindungen als die finnische Gemeinde verfügte, die wohl fast ausschließlich aus einfachen, weniger kapitalkräftigen Leuten bestanden haben dürfte (Murray, 1954: 18).

Die Kapelle erwies sich jedoch als zu klein für zwei Gemeinden und im März 1594 beschwerte sich die deutsche Gemeinde bei König Sigismund, dass sie nicht über eine eigene Kirche verfügte und brachte den Wunsch vor, die St.-Gertruds-Kapelle alleine nutzen zu dürfen, die sie zur Kirche ausbauen lassen wollte (die oben erwähnte Bittschrift abgedruckt bei Lüdeke, 1823: 68-72). Das Schreiben blieb unbeantwortet, aber am 21. Januar 1607 erhielt die deutsche Gemeinde das alleinige Nutzungsrecht der St.-Gertruds-Kirche durch einen offenen Brief König Karls IX. Eine Woche zuvor hatte der König der finnischen Gemeinde in einem offenen Brief die ehemalige Klosterkriche auf Gråmunkeholm zugewiesen.

Weitere Um- und Ausbauten der St.-Gertruds-Kirche fanden Mitte der 159oer Jahre statt. Offenbar im Zusammenhang mit diesen Verbesserungsarbeiten am Kirchengebäude hielt sich auch der Verfasser des hier zu besprechenden Briefes, der Dachdecker Mattewes Schroder, in Stockholm auf. Diesen Aufenthalt erwähnt er am Ende seines Briefes (s. Abschnitt 3.1, 3.2).

Edition und graphematische Analyse von Mattewes Schroders Brief an den Rat der Deutschen St. Gertruds Gemeinde zu Stockholm

Der Brief ist im Archiv der Deutschen St. Gertruds Gemeinde zu Stockholm („Tyska S:ta Gertruds kyrkoarkiv“) im Stockholmer Stadtarchiv („Stockholms stadsarkiv“) im Band „Skrivelser till kyrkorådet, äldre serie“ (SE/SSA/oo17/ J II/1/177-180) aufbewahrt. Nach der eigentlichen Edition in Form eines vollständigen, diplomatischen Transkripts sowie von Fotografien der beiden Textseiten in Abschnitt 3.1 folgen in Abschnitt 3.2 Angaben zu Aufbau und Inhalt des Briefes sowie in Abschnitt 3.3 Kommentare zur äußeren Form und graphematischen Besonderheiten. 


\subsection{Transkript des Briefes}

Der Brief ist zeilengetreu transkribiert, das Zeilenende ist zusätzlich durch einen Schrägstrich ,/‘ mit führendem Leerzeichen gekennzeichnet, das Seitenende durch einen doppelten Schrägstrich ,//' (da der Brief ansonsten keinerlei Interpunktionszeichen enthält, ist dies unzweideutig). Das interlinear ergänzte nicht in Z. 14 ist durch 'nicht' gekennzeichnet, der Nasalstrich wurde aufgelöst und ist durch kursiviertes $n$ wiedergegeben, Ergänzungen von mir (NJR) sind durch eckige Klammern gekennzeichnet. Für weitere Kommentare sei auf Abschnitt 3.3 unten verwiesen.

Der Brief umfasst 46 Textzeilen auf den Seiten 177 und 178 sowie eine vierzeilige Adresse und eine dreizeilige, schwer zu entziffernde Eingangsnotiz auf Seite 180. Die Fotografien in Abb. 1 und 2 unten zeigen die beiden Textseiten 177 und 178.

177, 1 Lauß deo adi den 7 Marzi Anno 16o2 /

2 Mÿnen frundtliken grus vnde weiligen dienft ftedes zuuorn Wen Idt /

3 iuw wolginge wer mi fer leib zu horen vor mine perfone da[n]cke /

4 ick godt vor leibes gefundtheit So lange alß idt den heren behaget /

5 Ick dancke iuw vor alle gude woldat de gi an mi ertoget hebben $=$ /

6 das fuflange allen guden borgers den ick gearbeit hebbe gude $=1$

7 betalinge mi gedan hebben So begere gi mi wol wedder an Iuw[er] /

8 arbeit alfo mi eine gefelle berichtiget hefft dat ick iuw ock wol arbei=/

9 den wolde wen gi mi willen ferner gude betalinge don van wegen /

10 des geboutes fo hefft mi de gefelle alfo berichtiget dat dar ftene /

11 nodich weren fo kan ick nich vp den beschet[?] fodane arbeit maken /

12 gi moften erften hebben den ften vnde wen wi de ftene moften /

13 hebben fo mote wi de lengede vnde de brede [wissen?] den alle ften de fchick /

fick `nicht' menniger gegen ein ander wente dar is menniger form fo / moten de ftene hir erft gleifuret fin men kan fe nicht er glei= / furen erem de rechte mat van den ftenen hefft So weth ick / wol wat an den geboute nodich is fo moften wi den ock noch ander / ftene hebben dat heten holfften vnde eine tunne fpar kalck vnde / fo moften wi ock weten van den arbeideslohne wat gi mi dar vor / wolden thokefen den ick mufte fulf ander vp dat arbeit kom[?]en / den it Is [ausgestrichen: fer] dep van dacke vnde mofte ock hebben hulpe 2 van / Iuw en dacloner volck de mi kalk vnde ften vnde ftelgen $n$ / maken vnde den kalck vnde ften thodregen den dat dack dat / moth gans vmme gelecht fin vnde de ene fi ende moth gans / 


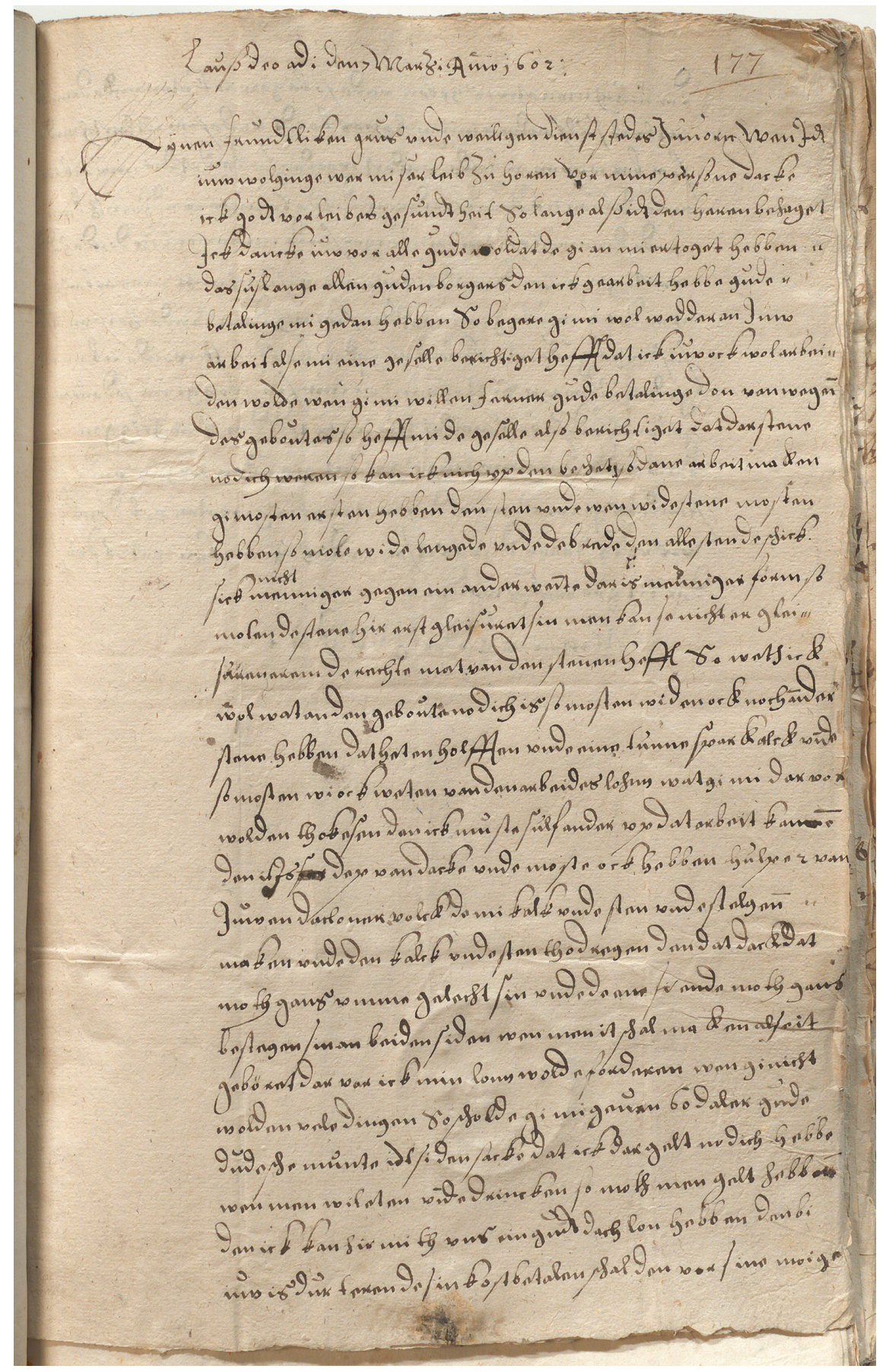

Авв. 1 Mattewes Schroders Brief, erste Textseite (SE/SSA/oo17/J II/1/177)

FOTO: STOCKHOLMS STADSARKIV 


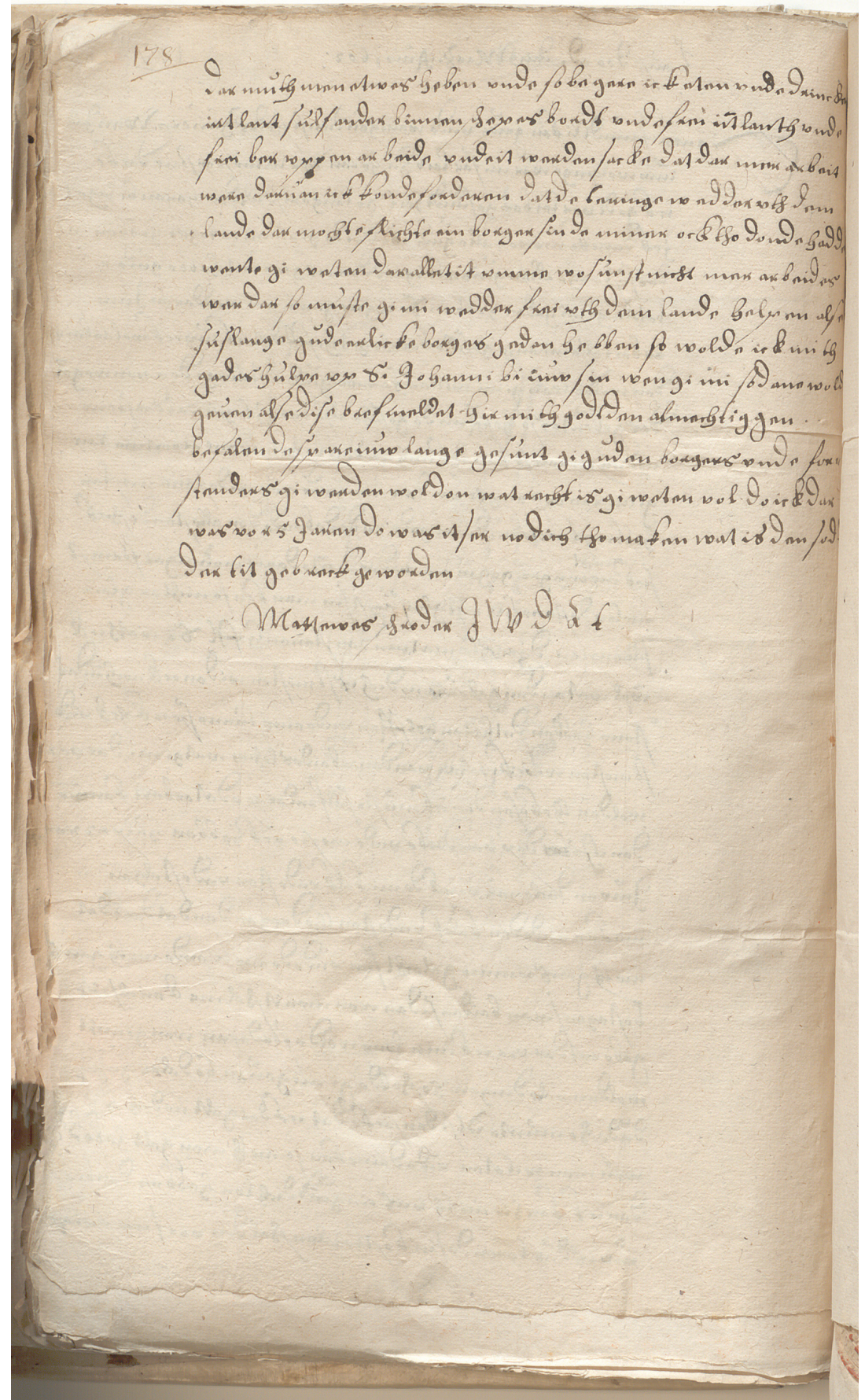

ABB. 2 Mattewes Schroders Brief, zweite Textseite (SE/SSA/oo17/J II/1/178) FOTO: STOCKHOLMS STADSARKIV 
25 beftegen fin an beiden fiden wen men it fchal maken alfeit /

26 geböret dar vor ick min lonn wolde forderen wan gi nicht /

27 wolden vele dingen So fcholde gi mi geuen 6o daler gude /

28 dudefche munte idt fi den facke dat ick dar gelt nodich hebbe /

29 wen men wil eten vnde drincken fo moth men gelt hebben /

3о den ick kan hir mith vns ein gudt dachlon hebben den bi /

$3^{1} \quad$ iuw is dur teren de fin koft betalen fchal den vor fine moige //

178, $3^{2}$ dar muth men etwas heben vnde fo begere ick eten vnde drinken /

33 int lant fulf ander binnen fchepes bordt vnde frei int lanth vnde /

34 frei ber vppen arbeide vnde it werden facke dat dar mer arbeit /

35 were daruan ick konde forderen dat de teringe wedder vth dem /

$3_{3} \quad$ lande dar mochte flichte ein borger fin de miner ock tho donde hadde /

37 wente gi weten dar allet it vmme wo funft nicht mer arbeides /

$38 \quad$ wer dar fo mufte gi mi wedder frei vth dem lande helpen alfe /

39 fuflange gude erlicke borge[r]s gedan hebben fo wolde ick mith /

40 godes hulpe vp S. Iohanni bi iuw fin wen gi mi fodane wolden /

${ }_{41}$ geuen alfe dife bref in[h]eldet hir mith godt den almechtiggen /

42 befalen de fpare iuw lange gefunt gi guden borgers vnde for- $/$

43 ftenders gi werden wol don wat recht is gi weten vol [wol] do ick dar /

44 was vor 5 Iaren do was it fer nodich tho maken wat is den fodd /

45 der fit gebreck geworden /

46 Mattewes fchroder I W D a t //

180, 47 [Adresse, auf dem Kopf:]

Ann de Erfamen borger vnde vor $=1$

48 ftender der dudefchen karken binnenn /

49 ftockholm kome diffe breff tho /

5o handen Rgfch /

$5^{1} \quad$ [Eingangsnotiz, von anderer Hand, nicht auf dem Kopf:]

Anno [1]6o2 den 12 Martzi /

$5^{2}$ kam thea[?] vns danken Empfangen /

53 [?]teggen[?] Der gemeine // 


\subsection{Zum Aufbau und Inhalt des Briefes}

Bei dem vorliegenden Schreiben Mattewes Schroders handelt es sich um einen Brief im Sinne der von Golz (2007: 251) gegebenen Definition: Der Text ist eine schriftliche Mitteilung, die von einer Einzelperson verfasst wurde (hier Mattewes Schroder) und an eine bestimmte, genannte und abwesende Gruppe von Personen gerichtet war (hier an den Ältestenrat der deutschen Gemeinde zu Stockholm) und nicht zur weiteren Veröffentlichung bestimmt war. Dementsprechend folgt der Brief auch der von Meier dargestellten Textstruktur mittelalterlicher und frühneuzeitlicher Briefe (siehe Tabelle 1).

TABELLE 1 Ideale vollständige Textstruktur der Kommunikationsform Brief

\begin{tabular}{|c|c|c|c|c|}
\hline & Textteile & & $\begin{array}{l}\text { Elemente der } \\
\text { Textstruktur }\end{array}$ & Inhalt \\
\hline I. & Protokoll & $\begin{array}{l}1 . \\
2 .\end{array}$ & $\begin{array}{l}\text { Salutatio } \\
\text { Exordio bzw. Captatio } \\
\text { benevolentiae }\end{array}$ & $\begin{array}{l}\text { Gruß, Anrede } \\
\text { Begründung des Briefes bzw. } \\
\text { Bitte um Gunst; Bemühen um } \\
\text { Wohlwollen, Aufmerksamkeit } \\
\text { und Interesse beim Empfänger }\end{array}$ \\
\hline II. & Substantia & 3. & Narratio & $\begin{array}{l}\text { Umstände; Darstellung des } \\
\text { Sachverhalts }\end{array}$ \\
\hline III. & Eschatokoll & $\begin{array}{l}4 . \\
5 .\end{array}$ & $\begin{array}{l}\text { Petitio } \\
\text { Conclusio }\end{array}$ & $\begin{array}{l}\text { Bitte; Vorbringen des Anliegens } \\
\text { Schlussworte (einschließlich } \\
\text { Grußformel; Datum und Tag) }\end{array}$ \\
\hline
\end{tabular}

QUELLE: MEIER, 2004: 170

Allgemein handelt es sich bei Mattewes' Brief um ein Angebot für auszuführende Dachdeckerarbeiten. ${ }^{5}$ Abweichend von der in Tabelle 1 gegebenen idealtypischen Grundstruktur beginnt er mit der Datumsangabe (Z. 1) statt mit ihr zu schließen. Danach folgen die Salutatio mit einer einleitenden Grußformel (Z. 2, s. auch Beispiel (2) in Abschnitt 4.2) sowie die Captatio benevolentiae mit Gesundheitsbekundungen und -wünschen um beim Empfänger Wohlwollen zu erwecken (Z. 2-7).

5 Dies ergibt sich aus den beschriebenen Arbeiten und benötigten Materialien, s. vor allem Z. 15, 18, 21, 23-25. 
Es folgt die Narratio (Z. 7-27), in der der Briefverfasser darauf zu sprechen kommt, dass ihm zugetragen worden sei, dass die Gemeinde in Stockholm seine handwerklichen Dienste nötig habe und dass er den Auftrag gerne annehmen wolle, sollte er dafür gute Bezahlung erhalten (Z. 7-9). Er beschreibt dann, welche Materialien und welche Arbeiten nötig sein werden sowie welchen Bedarf an Arbeitskräften er haben werde (Z. 10-26): Er benötigt Steine in der richtigen Größe, die zunächst glasiert werden müssen, es dürfte sich also um Dachziegel handeln. An weiterem benötigten Material nennt er noch Firstziegel (holfften, Z. 18) sowie eine Tonne Sparkalk (aus Gips gebrannter Kalk). An Arbeitskräften benötigt er zunächst noch eine weitere Person, die mit ihm zur Arbeit kommt (hier könnte es sich um seinen Gesellen handeln), weiterhin zwei Helfer von der Gemeinde sowie eine Tagelöhnerschar. In diesem Zusammenhang beschreibt er dann auch eindeutig Dacharbeiten: den dat dack dat / moth gans vmme gelecht fin vnde de ene fi ende moth gans / beftegen fin an beiden fiden (Z. 23-25).

Im Anschluss an die Beschreibung der nötigen Arbeiten und des benötigten Materials kommt Mattewes dann in der Petitio auch auf den Lohn zu sprechen, den er für sich selbst und zur Bezahlung der Arbeiter fordert: 6o Taler "gute deutsche Münze“ (gude dudesche munte, Z. 27 f.). Außerdem fordert er Verpflegung für die Reise (sowohl die Hin- als auch die Heimreise) sowie Freibier auf der Arbeit. Es fällt auf, dass die Petitio, die von der Bezahlung und Verpflegung handelt, etwas umständlich formuliert ist. Sie ist mit Höflichkeitsfloskeln und Ermahnungen an die Pflicht guter ehrlicher Bürger angereichert (Z. 36, 39), sie enthält Doppelungen des Gesagten (Z. 33; Z. 35-38), entschuldigende Hinweise darauf, dass er das Geld fordert, weil er es zum Leben nötig habe (Z. $28-32$ ) und die Lebenshaltungskosten in Stockholm so hoch seien (den bi iuw is dur teren de fin koft betalen fchal, Z. $3 \circ$ f.). Weiter enthält es unvollständige Sätze (Z. 35) und inhaltliche Sprünge (Z. 32-34 Verpflegung; Z. 34 f. Weitere Forderungen, sollte mehr Arbeit nötig sein; Z. 35 f. Verpflegung). Insgesamt macht das Textstück den Eindruck, als sei es dem Verfasser schwer gefallen, die Forderungen im Spannungsfeld zwischen Schicklichkeit und Notwendigkeit adäquat zu formulieren.

In der Conclusio, zum Abschluss des Briefes, schreibt er, dass er zu St. Johannis in Stockholm sein könne, sollten die Gemeindevorsteher das Angebot annehmen. Daran schließt eine Abschiedsformel an (hir mith godt den almechtiggen befalen [,] de spare iuw lange gesunt [.], Z. 41 f.). Im Anschluss an die Abschiedsformel kommt er dann aber nochmals darauf zu sprechen, dass er schon fünf Jahre zuvor in Stockholm gewesen sei und dass schon damals eine nicht näher spezifizierte Arbeit fällig gewesen wäre, vermutlich 
an einem Dach, das nun zwischenzeitlich tatsächlich schadhaft geworden sei (Z. 44 f.). Durch seine Positionierung nach der Abschiedsformel fällt dieser Satz besonders ins Auge und man kann vermuten, dass sich der Verfasser dadurch für die Arbeiten besonders empfehlen wollte.

Der Verfasser zeichnet seinen Brief mit Mattewes Schroder. ${ }^{6}$ Aus dem Inhalt des Briefes geht wie gezeigt hervor, dass er Dachdecker war. Dies bestätigt auch das Siegel, mit dem der Faltbrief verschlossen war, und auf dem sich das Zunftzeichen der Dachdecker - Dachdeckerbeil, Schieferhammer und Zirkel - erkennen lässt. Weiter lässt sich aus dem Brief erfahren, dass sein Verfasser nicht aus Stockholm selbst kam, nicht einmal aus Schweden, denn er spricht von seiner Schiffsreise ins Land und Heimreise wieder außer Landes, dass er aber mit den Stockholmer Verhältnissen zumindest in gewissem Grade vertraut war, hatte er sich doch schon fünf Jahre vor dem Verfassen des Briefes einmal dort aufgehalten und für die Gemeinde gearbeitet (Z. 44 f.). Woher Mattewes Schroder aber genau stammt, lässt sich leider nicht erfahren. Er macht in seinem Brief keine Ortsangabe und auch eine Durchsicht der Rechnungsakten der deutschen Gemeinde in Stockholm sowie derStockholmer Denkelbücher (,Stockholms stads tänkeböcker $\left.{ }^{6}\right)^{7}$ sowohl für die Zeit im Anschluss an den Brief als auch für einen ungefähr fünf Jahre zuvor liegenden Zeitraum, hat leider nichts eindeutiges ergeben, wobei das Denkelbuch für das Jahr 1598 nicht erhalten ist. Zwar machen die in den Rechnungsakten verzeichneten Ausgaben für beide Zeiträume ersichtlich, dass die Gemeinde Renovierungs- oder Bauarbeiten hat vornehmen lassen, doch weder ist eine Zahlung an einen Mattewes Schroder (in dieser oder ähnlicher Namensform) verzeichnet, noch findet sich eine entsprechende Verifikation aus seiner Hand.

Möglicherweise lassen sich aber doch einige Angaben mit ihm in Verbindung bringen. So sind für das Rechnungsjahr 1596/1597 Ausgaben für Dachdeckerarbeiten verzeichnet: „den dach laten mid nien stenen vth beteren“ (SE/SSA/oo17/L I ab/2/394). Betrachtet man die Aussage des Briefschreibers "gi

6 Im Text des Artikels verwende ich die Namensschreibung, die der Verfasser selbst in seiner Unterschrift verwendet hat - abgesehen von der Kleinschreibung des Familien- bzw. Beinamens (die sch-Ligatur findet sich im Brief nur in Kleinbuchstaben, gleichzeitig war die Kleinschreibung von Familiennamen zu dieser Zeit nicht ungewöhnlich, der Vorname wurde noch als der eigentliche Name betrachtet). Es lässt sich vermuten, dass der Familienname Schröder gelautet haben dürfte, Umlaut wird aber im Brief generell nicht konsequent und nur an einer Stelle gekennzeichnet (geböret, Z. 26), während er an anderer Stelle ungekennzeichnet bleibt (etwa mosten, Z. 12); siehe auch Transkription des Briefes in Abschnitt 3.1.

7 Rechnungsakten: „Räkenskaper för kyrka och församling. Äldre serie, original“, SE/SSA/oo17/L I ab; Denkelbücher: Stockholms stadsarkiv (1954); Stockholms stadsarkiv (1957). 
weten vol [wol] do ick dar was vor 5 Iaren do was it ser nodich tho maken wat is den sodder sit gebreck geworden" (Z. 43-45), lässt sich spekulieren, dass er bei den in den Rechnungsakten verzeichneten Ausbesserungsarbeiten anwesend oder beteiligt war und schon zu diesem Zeitpunkt darauf hingewiesen haben mag, dass eigentlich eine umfassendere Renovierung des Daches nötig wäre. Auch die Kosten der Ausbesserungsarbeiten aus dem Jahr 1596/97 liegen mit insgesamt 18 Talern und 21 Öre deutlich unter den im Brief geforderten 6o Talern, was - immer gesetzt den Fall, dass es sich tatsächlich um Arbeiten am selben Dach handelt - den größeren Umfang der vom Briefschreiber für nötig erachteten Arbeiten zeigt. Da aber weder bei den Ausgaben selbst noch unter den Verifikationen der Name Mattewes Schroder begegnet, muss dies letztlich Spekulation bleiben. Dennoch sollten diese - wenn auch vagen Beobachtungen anhand der Rechnungsakten hier nicht vorenthalten werden.

Auch ein Versuch, die Herkunft des Briefschreibers über das Wasserzeichen im Briefpapier einzugrenzen, ist bislang leider erfolglos geblieben. Und so lässt sich über die spärlichen im Brief gegebenen und aus dem Brief geschlossenen Angaben hinaus leider wenig über ihn erfahren.

Aus der Tatsache schließlich, dass er den Brief auf Niederdeutsch geschrieben hat, lassen sich noch einige Rückschlüsse ziehen. So kann man vermuten, dass er das Niederdeutsche als Muttersprache gehabt haben dürfte, vor allem aber, dass er seine schriftsprachliche Sozialisation in dieser Sprache erfahren hat. Geht man davon aus, dass Mattewes eine Schule besucht hat, so kann man zwar nicht sagen, wo und wann genau, es lässt sich jedoch vermuten, dass dies in den 158oer Jahren oder sogar noch früher geschehen ist, da er im Jahr 1602 Dachdecker, wahrscheinlich Dachdeckermeister ist. Zu dieser Zeit waren die Schulen in Norddeutschland noch Niederdeutsch; Sodmann (2000: 1507) datiert den Übergang der Schulen vom Nieder- zum Hochdeutschen auf den Anfang des 17. Jahrhunderts. Auch Gabrielsson (1932-1933: 78 f.), der von einer 150-jährigen Übergangsperiode ausgeht, verlegt den endgültigen Übergang für die meisten Gebiete ins 17. Jahrhundert (mit Ausnahme der unmittelbar mitteldeutschem Einfluss ausgesetzten Gebiete).

\subsection{Zur äußeren Form und graphematischen Besonderheiten des Briefes}

Der Brief findet sich auf einem Faltbriefbogen, die eine Hälfte des Bogens ist auf der Vorderseite vollständig beschrieben, auf der Rückseite zur Hälfte. Auf der anderen Hälfte des Bogens, die im gefalteten Zustand nach außen wies, finden sich Adresse und Siegel sowie eine Eingangsnotiz von anderer Hand. Einschließlich der Datumszeile am Anfang des Briefes sowie der Unterschrift am Ende umfasst der Brief insgesamt 46 Textzeilen und vier Adresszeilen. 
Der Brief ist in insgesamt gut leserlicher, recht aufrechter Schrift geschrieben, wobei einige Buchstaben durchgehend deutlich rechtsschräg erscheinen, namentlich $\langle f, \beta, p\rangle$, teils auch $\langle\mathrm{k}\rangle$. In einem der drei Fälle, in denen Mattewes Schroder den Buchstaben $\langle\mathrm{z}\rangle$ verwendet, zuuorn (Z. 2), ist dieses Zeichen leicht linksschräg. Weiterhin fällt bei allen drei $\langle z\rangle$-Schreibungen auf, dass der Buchstabe mit Ober- und Unterlänge geschrieben ist. Auch wenn dies auch für den Kleinbuchstaben durchaus nicht unüblich ist, so erweckt doch die ausladende Form bei Mattewes eher den Anschein eines Großbuchstaben, obwohl es sich in allen drei Fällen um den Kleinbuchstaben handeln dürfte: Marzi (Z. 1), zuuorn (Z. 2), zu (Z. 3). Die Auffälligkeiten bei der Schreibung des $\langle\mathrm{z}\rangle$ lassen sich möglicherweise dadurch erklären, dass Mattewes im Schreiben dieses vor allem im Hochdeutschen begegnenden Buchstabens ungeübt war.

Erwähnenswert ist in diesem Zusammenhang einzelner Graphemformen vielleicht auch noch das $\langle\mathrm{o}\rangle$, wenn es vor einem Graphem steht, das auf der Grundlinie beginnt. Dann kann das $\langle\mathrm{o}\rangle$ nämlich eine Form erhalten, die stark einem $\langle a\rangle$ ähnelt (muss es aber nicht): etwa in godes (Z. 57), nicht aber in godt (Z. 4, 41), wo das $\langle\mathrm{d}\rangle$ nicht auf der Grundlinie beginnend geschrieben ist, weiter vor $\langle\mathrm{m}\rangle$ in komen (Z. 20) sowie kome in der Adresse (Z. 49), nicht aber vor $\langle\mathrm{n}\rangle$ in don (Z. 9, 43), donde (Z. 36) usw., wo zwischen $\langle\mathrm{o}\rangle$ und $\langle\mathrm{n}\rangle$ die Feder deutlich abgesetzt wurde. Dass es sich bei diesen Schreibungen um eine graphematische Variante des $o$ handelt, die vor Buchstaben, die auf der Grundlinie beginnen, stehen kann, und nicht um die sich im Laufe des Mittelniederdeutschen durchsetzende Schreibung von $a$ für $o$ (vgl. Lasch, 1914: $\S 89$, S. 65 f.), wird durch das Nebeneinander beider Varianten in godt und godes deutlich.

Mattewes verwendet kaum Abbreviaturen, allein der Nasalstrich begegnet spärlich, und zwar hauptsächlich in Wörtern, die am Zeilenende stehen: ${ }^{8}$ wegen (Z. 9), annder (Z. 17), vnde (Z. 18), kom[?]en (Z. 20), ftelgenn (Z. 22), hebben (29), binnenn (48). Im Zeileninnern erscheint der Nasalstrich nur in vier Fällen: Anno 1602 (Z. 1), wente (Z. 14), vnde (Z. 29), int (Z. 33).

Am Zeilenende lässt sich der Nasalstrich damit erklären, dass der Schreiber Platz sparen wollte, um das Wort vollständig auf die jeweilige Zeile schreiben zu können mit Ausnahme des doppelten $n$ in ftelgenn, Z. 22, das zwar auch am Zeilenende steht, es dort aber genügend Platz gegeben hätte (s. Abb. 1 in Abschnitt 3.1). In den drei Fällen außerhalb der teils lateinischen Datumsangabe (Anno, Z. 1), wo der Nasalstrich in Wörtern verwendet wird, die nicht am Zeilenende stehen, steht er entweder vor einem $t$ (wente, Z. 14; int, Z. 33) oder einem $d$ ( $v$ nde, Z. 29). Vielleicht lässt sich der Nasalstrich hier

8 In den kursivierten Beispielen ist das aus dem Nasalstrich aufgelöste $n$ recte gesetzt. 
als Verbesserung einer Fehlschreibung erklären, die darin bestand, dass der Schreiber ein $n$ zwar begonnen, dann aber nicht vollständig zu Ende geführt hat, was er dann nachträglich als falsch oder zumindest unvollständig und korrekturbedürftig empfunden habe könnte. Dass von den 19 Fällen von vnde 17 ausgeschrieben sind und nur zwei durch Nasalstrich verkürzt, zeigt jedenfalls deutlich, dass Mattewes diese Abbreviatur nicht regelmäßig sondern eben nur unter bestimmten Bedingungen verwendet hat (etwa um Raum zu sparen oder zur Korrektur).

Abschließend sei hier nun noch auf einen Zug der Handschrift Mattewes Schroders in Bezug auf die Getrennt- und Zusammenschreibung von Wörtern und einzelnen Graphemen eingegangen, was letztlich auch für die sprachliche Deutung des Textes relevant ist. Zwar gilt grundsätzlich für frühneuzeitliche Texte, dass die Gründe für eine uneinheitliche Getrennt- und Zusammenschreibung sehr vielfältig sein können, etwa lassen sich Lücken innerhalb eines Wortes oft durch ein Absetzen der Feder, um neue Tinte aufzunehmen, erklären und unorganische Zusammenschreibungen können die Folge eines unachtsamen Neuaufsetzens der Feder sein (Bein, 1998: 926). Bei Mattewes' Handschrift fällt allerdings auf, dass er häufig Buchstabe für Buchstabe einzeln und mit kleinen Zwischenräumen geschrieben hat, obwohl die einzelnen Grapheme die Form einer Kurrentschrift haben. Nur solche Buchstabenfolgen, die schreibtechnisch so ineinander übergehen, dass sie geschrieben werden konnten, ohne dass die Feder vom Papier gehoben werden musste, hat er verbunden. Im ersten Satz des Briefes, also der Salutatio, handelt es sich hier beispielsweise um die Buchstabenfolgen nen, rund, en, nd, eili, ien, ed, es, uu, rn: Mÿ nen frund t li k en grus $v$ nd e w eili g en d ien ft ft ed es zuи orn (Z. 2).

Das gleiche gilt auch für eine Reihe kurzer, meist einsilbiger Wörter, bei denen sämtliche Buchstaben verbunden wurden: alle (Z. 13), allen (Z. 6), allet (Z. 37) an (Z. 5, 17, 25), de (Z. 5), ein (Z. 14, 30, 36), eine (Z. 8, 18), en (Z. 22), ene (Z. 24), er (Z. 15), erem (Z. 16), grus (Z. 2), idt/it (Z. 2, 4, 21, 25, 34, 37, 44), int (Z. 33), is (Z. 14, 17, 21, 31, 43, 44), iuw (Z. 5, 8, 22, 31, 40, 42), lant $(h)$ (Z. 33 zweimal), lonn (Z. 26), mat (Z.16), men (Z. 15, 29 zweimal, 32), mer (Z. 34), mi (Z. 3, 5, 7 zweimal, 8, 9, 10, 19, 22, 27, 38, 40), min (Z. 26), mine (Z. 3), miner (Z. 36), nich $(t)(\mathrm{Z} .11,14,15,26,37), f i(\mathrm{Z} .24)$, fo (Z.10, 11, 13, 14, 17, 19, 32, 38, 39), tho (Z. 36, 44, 49), wen (Z. 25).

Eine solche Schreibtechnik, die zu Zwischenräumen auch innerhalb von Wörtern führt, ist zwar nichts völlig ungewöhnliches und lässt sich auch bei anderen Schreibern beobachten, bei Mattewes führt sie aber dazu, dass es manchmal schwer wird, die Wortgrenzen zu erkennen, insbesondere da die Abstände innerhalb von Wörtern und zwischen Wörtern uneinheitlich groß 
sind. Dies kann wiederum zu Schwierigkeiten beim Textverständnis führen, nicht zuletzt auch, da Mattewes keinerlei Interpunktionszeichen verwendet: gimoft en erft en he $b b$ en d en ft en $v$ nd $e w$ en wi deft ene moft en / he b b en fo mo te wide leng ed ev nd e de b red e d en alle ft en defch ic $k / f i c k$ 'nicht' mennig ergeg en ein and erw ente d a is mennig erform (Z.12-14). Das Fehlen von Interpunktion ist in einem Brief, der um die Wende zum 17. Jahrhundert geschrieben wurde, natürlich nicht ganz unüblich und man sollte auch nicht den Fehler begehen, heutige Regeln der Zeichensetzung auf die noch offeneren syntaktischen Strukturen des frühneuzeitlichen Deutsch zu übertragen (Bein, 1998: 927). Nichtsdestotrotz wird hierdurch aber dem heutigen Betrachter das Verständnis des Brieftextes erschwert, insbesondere wo Sätze wie hier unvollständig bleiben bzw. ohne klare Trennung ineinander übergehen.

Anstelle von Interpunktionszeichen verwendet Mattewes in sechs Fällen einen großen Anfangsbuchstaben, um den Beginn eines neuen Satzes zu markieren. Dies gilt vor allem am Anfang des Briefes: Wen Idt iuw wolginge (Z. 2 f.), So lange alß idt den heren behaget (Z. 4), Ick dancke iuw (Z. 5), So begere gi mi wol (Z. 7), aber nur in zwei Fällen weiter unten im Text: So weth ick / wol (Z.16), So fcholde gi mi (27).

Darüber hinaus werden Großbuchstaben noch in einigen anderen Funktionen verwendet: In der Initiale im ersten Wort des Briefes: Mÿnen frundtliken grus (Z. 2), in Datumsangaben: Lauß deo adi den 7 Marzi Anno 1602 (Z. 1); vp S. Iohanni (Z. 40, zugleich Eigenname, s. u.), in einer Korrektur, die zu einem Tintenfleck führte: den it Is fer $\operatorname{dep}$ (Z. 21), als höfliche Anrede: 2 van Iuw (Z. 21 f.); Ann de Erfamen borger (Z. 47), im unpersönlichen Subjekt: Wen Idt iuw wolginge (Z. 2 f.), in einem Substantiv: vor 5 Iaren (Z. 44), bei Eigennamen und Titeln: $M$ att ew es fch ro der I WD a t (Z. 46) - jedoch nicht im Falle der $\langle f c h\rangle$ - und $\langle f t\rangle$-Ligaturen:fchroder (Z. 46); ftockholm (Z. 49).

\section{Codemixing in Mattewes Schroders Brief}

\subsection{Theoretischer Rahmen: Historisches Codemixing}

In der älteren sprachhistorischen Forschung wurde Phänomenen der Sprachmischung wenig Beachtung geschenkt. Nicht zuletzt vor dem Hintergrund einer in der Beschäftigung mit der Herausbildung der europäischen Nationalsprachen im 16. Jahrhundert gewonnenen Vorstellung, dass Einsprachigkeit die selbstverständliche Norm sei, wurden gemischtsprachige Texte als Ausdruck mangelnder Sprachkompetenz und Phänomene einer Übergangszeit auf dem Weg zum Sprachwechsel gesehen (Lüdi, 1985: 169; Schendl und Wright, 2011a: 17). Erst in Folge der Untersuchungen von Sprachmischung 
und Mehrsprachigkeit in der Gegenwartssprache sind diese Fragen dann seit Anfang der 199oer Jahre auch mehr und mehr in den Fokus der sprachhistorischen Forschung gerückt. Statt gemischtsprachige Texte als ,Unfall einer Übergangsperiode abzutun, wurden diese nun als früheste Beispiele von schriftlichem Codemixing betrachtet, die Aufschluss über Sprachgebrauch und sprachliche Strategien mittelalterlicher und frühneuzeitlicher Sprachbenutzer geben können (Schendl und Wright, 2011a: 17).

Die theoretischen und methodischen Grundlagen der Erforschung von Sprachmischung wurden allerdings seit Mitte der 196oer Jahre und zunehmend seit den 198oer Jahren in der Beschäftigung mit Phänomenen des Codemixings vor allem in der gesprochenen Sprache von Immigrantengruppen gewonnen (vgl. Heller und Pfaff, 1996: 594 f.; Stell, 2019: 159 f.). Dessen muss man sich bewusst sein, wenn man solche Ansätze auf historisches, schriftsprachliches Material übertragen will und so setzt auch die vorliegende Studie voraus, dass man die hermeneutische Grundannahme, dass eine solche Übertragung zulässig ist, akzeptiert (vgl. Kämmerer, 20o6: 36 f.). Einerseits kann, so Argenter (2001: 379), der neue, historische Blickwinkel zu neuen Einsichten und Erkenntnissen sowohl für die gegenwartssprachliche als auch die historische Betrachtung des Phänomens der Sprachmischung führen. Dies setzt aber andererseits aufgrund des fehlenden Zugriffs auf zeitgenössische, kompetente Sprecher sowie auf den situationellen und soziokulturellen Kontext der Kommunikationssituation der historischen Äußerung voraus, dass für die heutige linguistische Analyse dieser Kontext aus den überlieferten Texten selbst heraus und unter Zuhilfenahme von sprach-, kultur- und sozialhistorischem Wissen möglichst sorgfältig rekonstruiert werden muss (Argenter, 2001: 379, 381; Kämmerer, 2006: 37 f.). Dies bringt aber wiederum mit sich, dass alle Annahmen über die historische Kommunikationssituation und alle Ergebnisse einer Analyse des historischen Materials grundsätzlich vom verfügbaren historischen Wissen und von den theoretischen Grundannahmen des jeweiligen Betrachters gefärbt sind (vgl. Schendl und Wright, 2011a: 25).

\subsubsection{Formen des Codemixing nach P. Muysken}

In der Literatur wurden für Phänomene der Sprachmischung die verschiedensten Begriffe vorgeschlagen, wobei sich vor allem ,Codeswitching', aber auch ,Codemixing' als die meist-benutzten herauskristallisiert haben (Clyne, 2003: 70-76; vgl. Schendl und Wright, 2011a: 23). Da die Analyse im vorliegenden Beitrag vor allem auf den Überlegungen von Muysken (2000) aufbaut, wird dementsprechend auch weitestgehend die von ihm vorgeschlagene Terminologie (in deutscher Übersetzung) verwendet. Abweichend von Muysken (2000: 1) wird hier als allgemeiner Überbegriff nicht das von ihm 
vorgeschlagene und sporadisch verwendete „language interaction“ verwendet, sondern schlicht ,Sprachmischung“ oder ,gemischtsprachiger Text', während jede Art von Sprachmischung innerhalb eines Satzes, worauf sich Muysken (2000: 1) konzentriert, ihm folgend als,Codemixing bezeichnet wird.

Muysken (2000:3) unterscheidet drei Muster von satzinternem Codemixing, die er Insertion (engl. ,insertion'), Alternation (engl. ,alternation') und kongruente Lexikalisierung (engl. ,congruent lexicalization') nennt. Damit integriert er drei frühere Ansätze in sein Verständnis von Codemixing, die vor ihm teils im Gegensatz zueinander verwendet wurden. Unter Insertion versteht Muysken (2000: 3) das Einfügen von sprachlichem Material wie lexikalischen Einheiten oder Phrasen aus einer Sprache in die Struktur einer anderen Sprache, der sogenannten Matrixsprache. Hier greift er das von Myers-Scotton (1993) entwickelte ,Matrix Language-Frame Model' auf.

Im Gegensatz zur Insertion, bei der die zugrundeliegende Struktur die gleiche bleibt, also kein Sprachwechsel im eigentlichen Sinne passiert, ist Alternation gerade von einem solchen Sprachwechsel gekennzeichnet, also durch den Wechsel von einer Sprache in eine andere an einem Angelpunkt, wobei sowohl grammatische Struktur als auch lexikalische Elemente betroffen sind. Allein für diese Form des Codemixings ist laut Muysken (2000: 4) der andernorts oft als Überbegriff gebrauchte Terminus ,Codeswitching، angemessen. Unter dem Begriff Alternation versteht Muysken (2000) das von Poplack (1980) entwickelte Verständnis von Codeswitching (Muysken, 2000: 3-5).

Mit dem Begriff der kongruenten Lexikalisierung schließlich bezeichnet Muysken (2000) einen Codemixing-Prozess, bei dem Material aus dem lexikalischen Inventar verschiedener Sprachen oder Codes in eine geteilte grammatische Struktur eingefügt werden. Da eine solche geteilte grammatische Struktur, die keinem der beteiligten Codes eindeutig zugerechnet werden kann, wahrscheinlicher wird, je näher die Codes verwandt sind, lässt sich kongruente Lexikalisierung dementsprechend vor allem beim Wechsel der stilistischen Ebene oder bei Dialekt-Standard-Variationen beobachten, aber auch bei näher verwandten Sprachen. So hat etwa Clyne (1967) dieses Phänomen bei deutschen und niederländischen Immigranten in Australien beschrieben (Muysken, 2000: $3^{-6}$ ).

Da also, wie eingangs erwähnt, die strukturelle Nähe bzw. Ferne von Kontaktsprachen Codemixing im allgemeinen und kongruente Lexikalisierung im besonderen mehr bzw. weniger begünstigt, ${ }^{9}$ lässt sich erwarten, dass

9 Muysken (2000: 4, 16, 122 f.); vgl. dazu auch z. B. Kämmerer (2006: 12, 404, passim), die dies im Vergleich des Codemixings von Mittellatein mit Altitalienisch/Altspanisch bzw. mit Frühneuhochdeutsch beobachtet; außerdem: Clyne (2003: 162-179, insbes. 168, 177-179). 
Insertion längerer

Fragmente führt zu

zunehmend vollständiger

Aktivierung der zweiten

Grammatik

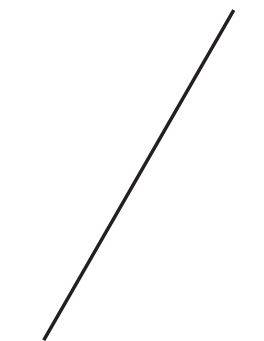

ALTERNATION
INSERTION

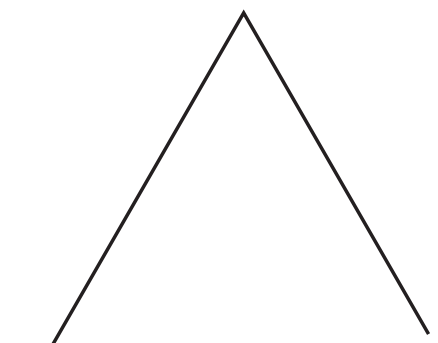

Übergang von einer

Grundsprache hin zu geteilter grammatischer Struktur durch zunehmende Annäherung der Strukturen der Kontaktsprachen in der Kontaktsituation

Grad der Überlappung der grammatischen Strukturen der Kontaktsprachen: inwieweit ist eine geteilte KONGRUENTE grammatische Struktur möglich?

Авв. 3 Schematische Darstellung der drei Hauptformen des Codemixings und der Übergänge zwischen ihnen VEREINFACHT NACH MUYSKEN, 2000: 9

dieser Prozess auch und gerade bei den beiden Kontaktsprachen Niederund Hochdeutsch zu beobachten sein dürfte, sind sie doch nicht nur nahe verwandt sondern sogar auf demselben (kontinental-westgermanischen) Dialektkontinuum zu verorten.

Die drei beschriebenen Formen des Codemixings sind nun nicht scharf voneinander abgegrenzte, sondern vielmehr graduell ineinander übergehende Phänomene, die man sich laut Muysken (200o: 9 f.) am einfachsten in einem Dreieck angeordnet vorstellen kann mit Übergangszonen zwischen Insertion und Alternation, Alternation und kongruenter Lexikalisierung sowie kongruenter Lexikalisierung und Insertion (s. Abb. 3). Die Insertion von längeren Fragmenten führt dazu, dass die Grammatik dieser Sprache mehr 
und mehr aktiviert wird, und sich so der (vollständigen) Alternation mehr und mehr annähert. Im Übergangsbereich von Alternation und kongruenter Lexikalisierung stellt sich die Frage, wie weitreichend die Überlappung der grammatischen Strukturen der beiden Kontaktsprachen ist und ob Sprachmischung also noch durch eine geteilte Struktur zu erklären ist oder nur durch einen Wechsel der grammatischen Struktur. Der Übergang von Insertion zu kongruenter Lexikalisierung ist durch eine Annäherung der Strukturen der Kontaktsprachen in der Kontaktsituation gekennzeichnet, die durch frequente Insertionen begünstigt wird und schließlich zu kongruenter Lexikalisierung und einer geteilten grammatischen Struktur führen kann.

Wie anhand der Analysen (Abschnitt 4.2) zu zeigen sein wird, eignet sich der integrative Ansatz Muyskens (2000) besonders gut, um die im vorliegenden Brief zu beobachtende Sprachmischung zu beschreiben. Die drei beschriebenen Formen des Codemixings werden nicht als einander entgegengesetzte und ausschließende Beschreibungsansätze von Sprachmischung verstanden, sonder als verschiedene, graduell ineinander übergehende Prozesse desselben Phänomens. Dieser graduelle Überganglässt sich auch im vorliegenden Material beobachten. Hinzu kommt noch, dass insbesondere der von Muysken (2000) beschriebene Prozess der kongruenten Lexikalisierung in einer leicht abzuwandelnden Form gerade zur Beschreibung der niederdeutsch-hochdeutschen Sprachmischung vor dem Hintergrund des Schreibsprachenwechsels adäquat erscheint, woraus sich die folgende Hypothese ergibt: Die hohe strukturelle Nähe der beiden Kontaktsprachen begünstigt diese Form des Codemixing und bringt mit sich, dass abweichend von Muyskens Reinform eine der beiden Kontaktsprachen das Niederdeutsche - im Prozess der kongruenten Lexikalisierung als geteilte sprachliche Struktur für beide Kontaktsprachen dienen kann.

\subsubsection{Kommunikative Funktionen von Codemixing}

Über die formale Analyse der Codemixing-Phänomene hinaus soll hier auch der Frage nach der Funktion des Codemixings in der historischen Kommunikationssituation nachgegangen werden, wie sie schon Lüdi (1985: 169) formuliert hat, warum nämlich der Schreiber in einer bestimmten Kommunikationssituation „zur Erfüllung seiner spezifischen Kommunikationsbedürfnisse gerade eine gemischtsprachige Äußerung anstelle einer einsprachigen“ formuliert. Zwar bietet auch Muysken (2000: 8 f.) Ansätze einer soziolinguistischen Interpretation, hier soll für die Interpretation der kommunikativen Funktionen des Codemixings jedoch auf Auer (1999) zurückgegriffen werden, der unter anderem davon ausgeht, dass Sprachmischung von den Kommunikationsteilnehmern soziale Bedeutung zugeschrieben wird, Auer (1999: 310) spricht hier von, konversationalem Codeswitching. 
Unter, Codeswitching fasst Auer (1999: 313 f.) nur Insertion und Alternation, während die kongruente Lexikalisierung nach Muysken (2000) für ihn wohl eher in den Bereich der Sprachmischung (,language mixing') fallen dürfte (vgl. Auer, 1999: 314). Unter konversationellem Codeswitching versteht Auer (1999: 310) einen diskursbedingten Wechsel in einen anderen Code, dem in der kommunikativen Situation eine bedeutungsvermittelnde Funktion zukommt. In Anlehnung an Muyskens (2000) Terminologie ließe sich hier aber auch von ,konversationellem Codemixing' sprechen, das dann alle drei Formen des Codemixing umfasst, denn Auer (1999: 318) weist auch darauf hin, dass eben nicht nur der Wechsel von einem Code in den anderen bedeutungstragend sein kann, sondern auch die Wahl eines sprachmischenden Modus („mixing mode“) selbst, im Gegensatz zu einem eher einsprachigen Modus. Wenn ein solcher sprachmischender Modus dann mit anderen sprachmischenden oder einsprachigen Modi kontrastiert wird, dann lässt sich bedeutungstragendes Codemixing auf mehreren Ebenen beobachten, also einerseits für die einzelnen Insertionen und Alternationen, andererseits für die Wahl des sprachmischenden Modus insgesamt (Auer, 1999: 318).

Vor dem Hintergrund der sich zunehmend etablierenden Konvention, dass das Hochdeutsche die Sprache der schriftlichen Außenkommunikation ist, kann sich ein in der niederdeutschen Schriftsprache sozialisierter Schreiber vor eine Herausforderung gestellt sehen, der er durch die Wahl eines sprachmischenden Modus als einer möglichen sprachlichen Strategie begegnen kann. Bei dieser sprachlichen Strategie dürfte es sich um eine gängige Kommunikationspraxis gehandelt haben. Dies zeigt schon der im zweiten Teil der Artikelserie zu den niederdeutschen Briefen an den Ältestenrat zu besprechende Brief des Hannß Bartteram, der vollständig in einem solchen sprachmischenden Modus geschrieben ist. Dass diese Praxis sich aber häufig wie hier bei Mattewes Schroder nur auf die Grußformel beschränkte (s. Abschnitt 4.2), belegen einige Hinweise in der älteren Literatur. So nennt wie oben erwähnt auch Gabrielsson (1983: 127) Anreden und formelhafte Wendungen unter den frühesten vom Hochdeutschen beeinflussten Textelemente. Entsprechend weist Lasch (1914: § 89, Anm. 2, S. 66, § 115, S. 79) darauf hin, dass gerade die Gruß- und Eingangsformeln von niederdeutschen Briefen und Urkunden für hochdeutschen Einfluss offen sind. Vor allem aber beobachtet auch Brattegard (1932: 279) in seinem Material der niederdeutschen Urkunden und Briefe des hansischen Kontors zu Bergen zahlreiche vollständig oder teilweise hochdeutsche Grußformeln in ansonsten niederdeutschen Briefen der Übergangszeit ab den 157 oer Jahren. Eine korpusbasierte, quantitative Studie, die genauere Angaben zur Häufigkeit dieser Praxis zuließe, liegt leider noch nicht vor und ließe sich als Forschungsdesiderat formulieren. 
In der hier vorliegenden Studie soll aber anhand einer qualitativen Analyse der Sprachmischung in der Grußformel von Mattewes Schroders Brief ein Beitrag zum Verständnis von Form und Funktion dieser sprachlichen Praxis gegeben werden.

Eine Mögliche Ursache für die Verwendung eines gemischtsprachigen Codes könnte dementsprechend auch darin liegen, dass der Schreiber dem intendierten Adressaten und Kommunikationspartner gegenüber bestimmte Signale senden möchte, wie etwa die Erfüllung einer angenommenen Erwartung. Die Bedeutung der Adressatenorientiertheit für die Sprachwahl bei ausgehenden Korrespondenzen zeigt auch Lüdi (1985: 173) am Beispiel der Missiven des Rates von Freiburg im Üechtland. Fürjeden Korrespondenzpartner wird immer die gleiche Sprache gewählt, die Schreiben selbst sind dann einsprachig.

Aus diesen Überlegungen ergibt sich die folgende Hypothese: Die Wahl des sprachmischenden Modus in der Grußformel trägt kommunikative Bedeutung und signalisiert Kommunikationsbereitschaft, denn sie spiegelt den kompetenten Umgang des Schreibers mit seinen sprachlichen Repertoires sowie sein Wissen um die Konventionen in der Kommunikationsform Brief sowohl hinsichtlich hochdeutscher Sprache als auch formalem Aufbau wieder.

\subsection{Analyse der Codemixing-Phänomene}

Beim ersten Betrachten der Codemixing-Phänomene in Mattewes' Brief könnte man auch hier, in die Falle tappen', in die schon die ältere Forschung gegangen ist, indem man als Hauptgrund für die Sprachmischung eine mangelnde Sprachkompetenz des Schreibers annimmt, der an dem Versuch scheitert, Hochdeutsch zu schreiben. Aber anhand der Analyse des Materials soll hier ein anderer Erklärungsansatz angeboten werden, der eher den kompetenten und bewussten Umgang des Schreibers mit seinen sprachlichen Repertoires vor dem Hintergrund des Übergangs von der nieder- zur hochdeutschen Schreibsprache ins Zentrum rückt als dessen mangelnde Kompetenz in einer der beteiligten Kontaktsprachen. Gleichzeitig soll unter Rückgriff auf Muyskens (2000) Modell auch noch ein geeigneterer Beschreibungsansatz für das Codemixing von nieder- und hochdeutsch in dieser besonderen historischen Situation gefunden werden.

Betrachtet man den vorliegenden Brief unter dem Aspekt der Sprachmischung, so darf man die Datumszeile (1) ganz zu Beginn des Briefes nicht unberücksichtigt lassen: Diese ist (fast ausschließlich) Lateinisch, so dass neben dem erwähnten Codemixing von Nieder- und Hochdeutsch hier also noch eine dritte Sprache verwendet wird. 


\section{(1) Lauß deo adi den 7 Marzi Anno 1602 (Z. 1)}

Da die vorliegende Untersuchung sich auf das Codemixing von Niederund Hochdeutsch konzentriert, soll hier nicht näher auf diese lateinische Datumszeile eingegangen werden, sie soll aber auch nicht unerwähnt bleiben, wirft sie doch ein Licht auf den kompetenten und bewussten Sprachgebrauch des Briefschreibers, der schon hier zeigt, dass er die formalen Konventionen des Briefschreibens kennt und ihnen folgen kann. Dafür spricht auch die Tatsache, dass Mattewes insgesamt der für seine Zeit gültigen konventionellen Textstruktur von Briefen folgt (s. Abschnitt 3.2).

Der Brief ist also insgesamt hauptsächlich auf Niederdeutsch verfasst, aber vor allem ganz am Anfang, in Salutatio und Captatio benevolentiae, finden sich einige hochdeutsche Elemente in abnehmender Frequenz: Im ersten Satz des Briefes sind von insgesamt acht Wörtern noch jeweils drei eindeutig hochbzw. niederdeutsch. Im zweiten Satz sind von zehn Wörtern zwei hochdeutsch, die restlichen Wörter sind niederdeutsch und im dritten Satz sind zwei von 16 Wörtern hochdeutsch. Im restlichen Brief treten hochdeutsche Wortformen nur noch vereinzelt auf.

(2) Mÿnen frundtliken grus vnde weiligen dienst stedes zuuorn (Z. 2)

Beispiel (2) gibt die Salutatio von Mattewes' Brief, in der die niederdeutschhochdeutsche Sprachmischung am augenfälligsten ist. Wie oben erwähnt sind formelhafte Wendungen, insbesondere Gruß- und Eingangsformeln nicht selten von hochdeutschem Einfluss und Sprachmischung betroffen. Allgemein handelt es sich bei diesem einleitenden Satz (2) um eine weitverbreitete Grußformel, die sich in ähnlicher Form in zahlreichen anderen spätmittelalterlichen und frühneuzeitlichen Briefen findet, und zwar sowohl auf Hochals auch auf Niederdeutsch. Auch Mähl (2008: 34) nennt die Formel „vnsen/ minen fruntliken grot tovoren“ als eine der häufigsten Briefeinleitungen in den Stockholmer mittelniederdeutschen Briefen (vgl. auch Kantola, 1983: 20). Zur Veranschaulichung seien hier noch einige Beispiele angeführt, wobei Satz (3a) hochdeutsch ist, Satz (3b) dem eingangs erwähnten, in niederdeutschhochdeutscher Mischsprache verfassten Brief des Hannß Bartteram entnommen ist und die Sätze (3c), (3d) und (3e), die im Vergleich zu dem hier behandelten Brief von Mattewes Schroder etwa hundert Jahre früher belegt sind, schließlich niederdeutsch sind.

(3) a. Meÿn frundtlich gruß nebenst wunschung zeitlicher vnd ewiger wolfarth zuuor (M. Petrus Hinkelman an den Ältestenrat der 
Deutschen St. Gertruds Gemeinde zu Stockholm, Rostock, 29.07.1601, SE/SSA/oo17/J II/1/175)

b. Mÿnen frundtlÿch grueß vnnd wÿllÿger dennst mÿdt wunschung Alles guden beuor (Hannß Bartteram an den Ältestenrat der Deutschen St. Gertruds Gemeinde zu Stockholm, Rostock, 28.07.16o1, SE/SSA/oo17/J II/1/171)

c. Minen gantz willigen und fruntlicken denst vor. (Johannes Patiner, Probst zu Dannenberg u.s.w. an Reval, 26.7.1505, Arbusow, 1905, Nr. 795, S. 618)

d. Unsen fruntliken grut myt vermoge alles guden stedes tovorn. (Hamm an Reval, 14.8.1505, Arbusow, 1905, Nr. 803, S. 622)

e. Mynen vruntliken gruith unde alle guith stedes thovoren. (Svante Nilsson [Sture], schwed. Reichsverweser, an Reval, 26.8.1505, Arbusow, 1905, Nr. 805, S. 823)

Eine detaillierte Analyse der in Beispiel (2) gegebenen Salutatio im Hinblick auf Codemixing zeigt, dass hier sowohl hoch- als auch niederdeutsches lexikalisches Material in eine von den beiden Kontaktsprachen geteilte sprachliche Struktur eingefügt werden, es liegt also kongruente Lexikalisierung vor. Von den einzelnen Wortformen sind Mÿnen, frundtliken und stedes eindeutig niederdeutsch, bei ersterem wäre hochdeutsch ein Diphthong im Stammvokal zu erwarten, bei zweiterem ein verschobenes ch im Suffix -lik/ -lich und bei letzterem ein verschobenes $t$, also das hochdeutsche Wort stets. Die Wortformen grus, dienst und zuuorn dagegen sind eindeutig hochdeutsch, ersteres und letzteres jeweils mit den durch die zweite Lautverschiebung zu erklärenden Reibelauten $s$ bzw. $z$ statt des niederdeutsch zu erwartenden Verschlusslautes $t$ und dienst schließlich mit langem $\bar{l}$. Auch weiligen dürfte hochdeutsch sein, bedarf aber einer näheren Betrachtung.

Die statt weiligen [dienst] zu erwartende Formulierung wäre willigen [dienst], so findet sie sich in der Salutatio zahlreicher frühneuzeitlicher Briefe, wie unter anderem auch hier in den Beispielen (3b) und (3c). Sollte vom Briefschreiber hier tatsächlich willigen dienst gemeint sein, dann ließe sich der Diphthong möglicherweise als Hyperkorrektur erklären: Er könnte die Regel, dass dem niederdeutschen langen $i$ im hochdeutschen der Diphthong $e i$ entspricht hier auch auf den Kurzvokal übertragen haben. Möglich wäre natürlich auch, dass er das hochdeutsche Wort willig an anderer Stelle gelesen und dann falsch erinnert hat, ohne dass eine bewusste oder unbewusste Identifikation von niederdeutsch $i$ mit hochdeutsch ei zu Grunde liegen würde. 
Schließlich kann auch nicht ausgeschlossen werden, dass der Schreiber tatsächlich weiligen meinte und zwar in der Bedeutung, allzeitig, stetig', womit es in der Formulierung hier eine Doppelung des folgenden stedes darstellen würde. Nach Auskunft der Wörterbücher ist weilig für das Frühneuhochdeutsche nicht belegt. Im Deutschen Wörterbuch (Grimm und Grimm, 1984) ist es nur mit der Bedeutung, ruhig' als jüngere Bildung des 19. Jahrhunderts verzeichnet, älter fände es sich nur als Zweitglied (etwa in derweilig usw.) und bei Adelung (2004) gibt es gar keinen Eintrag für weilig. Für das Mittelhochdeutsche ist jedoch laut Lexer (1992) das Adverb wîlec-lîche, -lîchen mit der Bedeutung ,zu jeder Zeit, zu jeder Stunde‘ belegt, das hier möglicherweise zu Grunde gelegt werden kann. Welche der beiden Erklärungen die richtige ist, lässt sich heute letztlich nicht mehr entscheiden. Sollte es die erstere sein, so haben wir hier mit der hyperkorrekten Form neben den Codemixing-Phänomen innerhalb von Satz (2) einen weiteren Ausdruck des Spannungsfeldes zwischen hochdeutscher Norm und niederdeutscher Sprachkompetenz, in dem sich der Briefschreiber bewegt.

Auch in den der Salutatio (2) folgenden ersten beiden Sätzen der Captatio benevolentiae (4) und (5) findet sich noch hochdeutsches lexikalisches Material, nun aber in geringerer Frequenz, so dass man hier eher von hochdeutschen Insertionen in die grammatische Struktur der niederdeutschen Matrixsprache sprechen kann. Der Übergang von alternierender Lexikalisierung hin zur Insertion und schließlich Einsprachigkeit im Brief ist demnach nicht abrupt sondern eher fließend entlang der abnehmenden Frequenz hochdeutscher Wortformen. In den Sätzen (4) und (5), die die obligatorischen Gesundheitswünsche und -bekundungen enthalten, findet sich noch je eine hochdeutsche Insertion, ansonsten sind sie auch lexikalisch vollständig niederdeutsch. Die folgenden ebenfalls zur Captatio benevolentiae zu zählenden Dankesformeln enthalten kein hochdeutsches Sprachmaterial mehr.

(4) Wen Idt iuw wolginge wer mi ser leib zu horen (Z. 2 f.)

(5) vor mine persone da[n]cke ick godt vor leibes gesundtheit So lange alß idt den heren behaget (Z. 3 f.)

In Satz (4) ist leib zu hochdeutsch, wobei es sich bei leib entweder um eine Fehlschreibung für lieb handeln könnte, um eine fälschliche Assoziation des niederdeutschen Langvokals $\bar{e}$ mit dem hochdeutschen Diphthong oder aber um eine Schreibung mit niederdeutschem Dehnungs- $i$, so dass es also als $l \bar{e} b$ 
auszusprechen wäre und dann eine Art Mischform aus niederdeutsch lēf und hochdeutsch lieb darstellen würde.

Im folgenden Satz (5) ist dann leibes gesundtheit hochdeutsch, niederdeutsch müsste es lïfes gesunt oder gesunde heißen. Hier ist im Schriftbild bemerkenswert, dass der Briefschreiber das Wort gesundt zunächst mit der $d t$-Ligatur scheibt, die er sonst nur am Wortende hat (in der Binnenposition schreibt er $d t$ nicht als Ligatur) und dann das Suffix -heit mit einem Zwischenraum dahintersetzt. Dies hinterlässt den Eindruck, der Schreiber habe möglicherweise zunächst das niederdeutsche gesundt geschrieben, um es dann zum hochdeutschen gesundtheit zu verbessern. Allerdings muss einschränkend gesagt werden, dass sich $d t$ im Brief nur noch an einer einzigen weiteren Stelle in der Binnenposition findet (frundtliken, Z. 2), dass die Abstände zwischen einzelnen Wörtern sowie innerhalb von Wörtern wie erwähnt recht uneinheitlich groß ausfallen können und dass die Getrenntschreibung von Stamm und Suffix insgesamt nicht ungewöhnlich ist, so dass der Abstand zwischen $d t$ und dem Suffix hier auch nicht überbewertet werden darf, immerhin weist er aber auf einen Neuansatz der Feder - aus welchem Grund auch immer - hin.

Nach den einleitenden Gruß- und Höflichkeitsformeln bleibt der Brief mit Ausnahme einiger weniger hier in (6) und (7) wiedergegebener Fälle vollständig Niederdeutsch: Bei diesen Ausnahmen handelt es sich dreimal um das hochdeutsche frei (Z. 33, 34, 38) statt niederdeutsch frī sowie um flichte (Z. 36) für niederdeutsch plicht. Alle vier Insertionen finden sich in der oben in Abschnitt 3.2 zusammengefassten Petitio des Briefes, die Bitte und Appell zur adäquaten Bezahlung enthält.

(6) a. vnde fo begere ick eten vnde drinken / int lant fulf ander binnen fchepes bordt vnde frei int lanth vnde / frei ber vppen arbeide (Z. 32-34)

b. wente gi weten dar allet it vmme wo funft nicht mer arbeides / wer dar fo mufte gi mi wedder frei vth dem lande helpen alfe / fuflange gude erlicke borge[r]s gedan hebben (Z. 37-39)

(7) daruan ick konde forderen dat de teringe wedder vth dem / lande dar mochte flichte ein borger fin de miner ock tho donde hadde (Z. 35 f.)

Das Wort frei, das in Mattewes' Brief nur in der diphthongierten Form verwendet wird, könnte auch dadurch zu erklären sein, dass es in dieser Form schon in den Sprachgebrauch des Verfassers übergegangen war, weist doch 
Gabrielsson (1983: 127) darauf hin, dass gerade die Umsetzung von $i>e i$ einer der frühesten hochdeutschen Einflüsse auf das Niederdeutsche in der ersten Phase des Schreibsprachenwechsels ist. Dann wäre frei aber eher als Entlehnung denn als Codemixing zu betrachten, in dem Sinne als es in das mentale Lexikon des Sprachbenutzers aufgenommen wurde (vgl. Muysken, 2000: 70$)^{10}$

Die Form von flichte in Beispiel (7) bedarf hier noch einiger Anmerkungen. In der hochdeutschen Form wäre im Anlaut eigentlich die Affrikate $p f \mathrm{zu}$ erwarten, die Form mit Frikativ $f$ findet sich jedoch in einigen ostmitteldeutschen Dialekten (und dem Jiddischen). Die niederdeutsche Form hat im Anlaut den Verschlusslaut $p$, bei der Schreibung im Brief handelt es sich aber um eine eindeutige $\langle f\rangle$-Schreibung, die Grapheme von $\langle f\rangle$ und $\langle p\rangle$ sind auch nicht zu verwechseln. Geht man also von einer hochdeutschen Form aus, lässt sich der frikativische Anlaut dialektal als ostmitteldeutsche Form erklären. Da die Herkunft des Briefschreibers unbekannt ist, lässt sich allerdings nichts über die nächste hochdeutsche Kontaktvarietät sagen, es ist aber durchaus vorstellbar, dass er die ostmitteldeutsche Form kannte. Genauso möglich ist aber auch, dass der niederdeutsche Schreiber mit der Schreibung der hochdeutschen Affrikate nicht vertraut war und sein $\langle\mathrm{f}\rangle$-Graphem für die Affrikate steht. Schließlich kann es sich bei der Schreibung mit Frikativ auch noch um einen mechanischen Ersatz von niederdeutsch $p$ mit hochdeutsch $f$ handeln, ohne dass die Feinheiten der Verhältnisse von niederdeutschem Verschlusslaut zu hochdeutschem Frikativ bzw. hochdeutscher Affrikate berücksichtigt oder gekannt wurden, so dass es sich also auch bei flichte um eine Art hyperkorrekte Form handeln könnte.

Insgesamt findet sich in Mattewes' Brief also nirgendwo ein klarer Switch von einer Sprache in die andere, also einer Alternation (sieht man von der Datumszeile ab). Vielmehr zeigt sich der fließende Übergang zwischen kongruenter Lexikalisierung und Insertion im Sinne des auch von Muysken (200o: 9 f.) beschriebenen graduellen Kontinuums zwischen diesen Mustern von Codemixing: Die Sprachmischung in der Salutatio lässt sich noch eindeutig als kongruente Lexikalisierung beschreiben, dann nehmen aber die hochdeutschen Elemente mehr und mehr ab, sie sind in den der Salutatio folgenden ersten Sätzen der Captatio benevolentiae zwar noch recht häufig, können hier aber schon an der Grenze zu Insertionen in eine niederdeutsche Struktur beschrieben werden. Im weiteren Verlauf des Textes verschwinden

10 Über den Unterschied zwischen (insertionalem) Codemixing und Entlehnung und ob diese überhaupt als verschiedene Phänomene zu betrachten sind, wurden in der Literatur allerdings verschiedene Ansichten vorgebracht (vgl. dazu z. B. Muysken, 200o: 69-81). 
sie bis auf wenige Ausnahmen von hochdeutschen Insertionen schließlich fast vollständig.

Einen Sprachwechsel im Sinne einer Alternation könnte man höchstens dann als einen Wechsel aus dem Hochdeutschen ins Niederdeutsche an der Grenze zwischen Salutatio (2) und dem ersten Satz der Captatio benevolentiae (4) sehen, wenn man unterstellt, dass der Schreiber den Brief oder zumindest die Salutatio tatsächlich auf Hochdeutsch beginnt (oder beginnen will), dann aber doch aufgrund mangelnder hochdeutscher Sprachkompetenz, die sich schon an einzelnen niederdeutschen Insertionen in Satz (2) zeigt, in seine offensichtlich als Schreibsprache erlernte Muttersprache Niederdeutsch wechselt.

Adäquater erscheint es jedoch, den ersten Satz (2) im Sinne der kongruenten Lexikalisierung zu beschreiben, wenn auch vielleicht im Übergangsbereich hin zur Insertion, mit einer zugrundeliegenden niederdeutschen grammatischen Struktur, die aber durch ihre Nähe und Überlappung mit einer hochdeutschen Struktur als eine gemeinsame funktionieren kann. Im Bewusstsein um die Konvention füllt dann der Schreiber diese niederdeutsche bzw. geteilte Struktur teilweise mit hochdeutschem lexikalischem Material. Im Anschluss an den ersten Satz findet dann zu Satz (4) hin auch kein Wechsel zwischen zwei distinkten sprachlichen Strukturen statt, vielmehr findet sich ein fließender Übergang zu einem eher insertionalen Codemixing von immer weniger hochdeutschem Material in eine sprachliche Struktur, die sich dann mehr und mehr als eine niederdeutsche erweist und schließlich auch lexikalisch weitestgehend einsprachig gefüllt wird. Damit lässt sich als Beschreibungsansatz der niederdeutsch-hochdeutschen Sprachmischung im vorliegenden Brief und vielleicht auch in der beschriebenen besonderen historischen Situation insgesamt eine (Übergangs-)Form der kongruenten Lexikalisierung anbieten, die davon ausgeht, dass die zugrundeliegende Struktur des gesamten Textes (also einschließlich des ersten Satzes) Niederdeutsch ist, dann aber aufgrund der strukturellen Nähe der beiden beteiligten Sprachen als geteilte Struktur für die zweite Kontaktsprache Hochdeutsch funktionieren kann.

\subsection{Ansätze zur Erklärung für das Codemixing bei Mattewes Schroder}

Auch wenn hier also auf struktureller Ebene keine klar zu verortende Alternation nach Muysken (2000) beobachtet werden kann, so fällt eben doch auf, dass für die Salutatio bedingt durch die kongruente Lexikalisierung ein anderer Sprachmodus als für den Rest des Briefes gewählt wird, so dass man ausgehend von Auer (1999) hier durchaus von konversationellem Codemixing im oben beschriebenen Sinne sprechen kann (s. Abschnitt 4.1.2). Dieser Wahl 
des sprachmischenden Modus für die Salutatio kommt also kommunikative Bedeutung zu, die etwa darin liegen könnte, dass der Verfasser des Briefes signalisieren möchte, dass er sich dessen bewusst ist, dass die Stockholmer deutsche Gemeinde in ihrer Außenkommunikation Hochdeutsch verwendet. Die Wahl des sprachmischenden Modus erscheint als sprachliche Strategie des im Niederdeutschen sozialisierten Schreibers, dieser Konvention gerecht zu werden. Somit ist diese Wahl adressatenorientiert. Auch die Insertionen in (7) und eventuell (6) gegen Ende des Briefes lassen sich als konversationales Codemixing verstehen, in dem sich die Adressatenorientiertheit von Bitte und Appell auch auf sprachlicher Ebene widerspiegelt.

Neben der Adressatenorientiertheit lässt sich auch noch ein weiterer Grund für die Wahl des sprachmischenden Modus in der Salutatio annehmen, nämlich eine Textsortenorientiertheit. Der Schreiber zeigt sein Bewusstsein darüber, dass die Sprache der Außenkommunikation der Kanzleien und in gewissem Maße auch der Kaufleute im gesamten niederdeutschen Sprachraum zu diesem Zeitpunkt schon Hochdeutsch war (Gabrielsson, 1983: 131 f., 147-150; zu den Kaufleuten vgl. Brattegard, 1932: 279 f.; 1945: 9; 1946, XII; 1963: 8 f.; Katara, 1936: 182-184; 1950: 39; Nesse, 2007: 425). Damit könnte dieser Wahl des den Brief einleitenden sprachmischenden Modus auch eine ähnliche konversationelle Funktion wie der Höflichkeitsformel als solcher zukommen. Die Wahl des Lateinischen in der Datumszeile, die Wahl der hochdeutsch-niederdeutschen Sprachmischung in der Salutatio, die Formelhaftigkeit der Grußformel selbst sowie die Befolgung der Textstruktur zeitgenössischer Briefe insgesamt erfüllen alle auch die Funktion, den vorliegenden Text in der Textsorte Brief einzuordnen und sind damit gleichzeitig auch wieder adressatenorientiert, denn sie erfüllen einerseits die Erwartungen, die ein möglicher (intendierter oder tatsächlicher) Rezipient an den Text hat und signalisieren andererseits dadurch gleichzeitig auch Kommunikationsbereitschaft.

Dass nicht die einzelnen Sprachwechsel bedeutungstragend sind, sondern die Wahl des sprachmischenden Modus insgesamt, zeigt, dass die in der Salutatio zu beobachtende Form der kongruenten Lexikalisierung nach der Definition Auers im Grenzbereich zu einer Mischsprache anzusiedeln ist. Den fließenden Übergang von Codemixing zu einer Mischsprache beschreibt Auer (1999: 309 f., passim) als einen Übergang von „codeswitching“ über „language mixing“ hin zu „fused lects“. Den Unterschied zwischen konversationellem "codeswitching“ und „language mixing“ definiert Auer (1999: 310) über die konversationelle Funktion des Nebeneinanderverwendens zweier Codes, während bei ersterem der einzelne Codewechsel von den Kommunikationspartnern als lokal bedeutungsvermittelnd aufgefasst wird, ist beim „language 
mixing "die Verwendung zweier Codes als ein wiederkehrendes Muster auf einer globalen, etwa identitäts-definierenden (Auer, 1999: 320) Ebene bedeutungstragend. Der Unterschied zwischen „codeswitching“ und „language mixing“ liegt also darin, wie die Kommunikationsteilnehmer die fraglichen Codes wahrnehmen und deuten und ist somit Gegenstand soziolinguistischer Interpretation (Auer, 1999: 310). Da der sprachmischende Modus in der Salutatio sich in einem ansonsten niederdeutschen Brief findet und auch kein klarer Sprachwechsel, sondern ein fließender Übergang über insertionales Codemixing hin zur niederdeutschen Einsprachigkeit beobachtet werden kann, lässt sich aber die Sprachmischung in der Salutatio adäquat als Codemixing beschreiben.

Erst auf einer zweiten Ebene der Sprachmischung lässt sich dann einerseits die kongruente Lexikalisierung im ersten Satz (2) sowie die folgenden Insertionen in (4) und (5) beschreiben. Anders als bei der diskursbedingten Wahl des sprachmischenden Modus haben wir es hier dann eher mit einem kommunikationsteilnehmer-bedingten Codemixing im Sinne Auers (1999:310) zu tun, das möglicherweise auch in der Sprachkompetenz des Briefschreibers begründet liegt.

\section{Zusammenfassung und Ausblick}

Das Spannungfeld zwischen niederdeutscher schreibsprachlicher Tradition und Kompetenz, wie sie dem Schreiber Mattewes Schroder wohl als Muttersprachler, sicherlich aber durch Schreibspracherwerb zukommt, und seinem Wissen um die hochdeutsche Konvention im externen Sprachgebrauch (nicht nur) der Stockholmer deutschen Gemeinde spiegelt sich im Codemixing von Nieder- und Hochdeutsch in seinem Brief wider. Es konnte gezeigt werden, dass dieses Codemixing Ausdruck des kompetenten Umgangs des Schreibers mit seinen sprachlichen Repertoires ist, der adressaten- und textsortenorientiert bewusst einen sprachmischenden Modus in der Salutatio wählt, wie er auch die Formelhaftigkeit der Salutatio selbst, der Datumsangabe und der Briefstruktur insgesamt kennt und umsetzt. Der Schreiber, der die sprachliche Situation in Stockholm aus seinem früheren Aufenthalt gekannt haben dürfte, wusste, dass sowohl der sprachmischende Modus als auch das Niederdeutsche dort ohne Hindernis verstanden werden würden.

Codemixing konnte als adäquates Beschreibungsmodell der in Mattewes' Brief zu beobachtenden Sprachmischung angewandt werden, und formal lässt sich das Codemixing in der Salutatio als eine Form der kongruenten Lexikalisierung im Übergangsbereich zur Insertion beschreiben, wo eine zugrundeliegende niederdeutsche Struktur mit hoch- wie niederdeutschem 
lexikalischem Material gefüllt wird. Diese Form der kongruenten Lexikalisierung beruht hier nicht darauf, dass der Schreiber in beiden Sprachen fast gleichermaßen kompetent ist und so die strukturelle Ähnlichkeit dieser beiden Sprachen in seinem Sprachgebrauch so nutzt, dass er sich aus dem lexikalischen Inventar beider Sprachen bedient. Vielmehr könnte es hier auch so sein, dass die Kompetenz, die der Schreiber in der ihm eigentlich fremden Sprache hat, (vor allem) auf derstrukturellen Nähe der beiden Kontaktsprachen beruht. Das heißt, dass die zugrundeliegende sprachliche Struktur die seiner niederdeutschen Muttersprache ist (also der Matrix-Sprache der Insertionen vergleichbar), die strukturelle Nähe zur Kontaktsprache Hochdeutsch macht es dann aber möglich, dass diese Struktur auch als grundlegende Struktur dieser zweiten Sprache dienen kann und mit lexikalischem Material aus beiden Sprachen gefüllt werden kann. Aber auch einen guten Teil des hochdeutschen lexikalischen Materials scheint der Schreiber dann nicht aus dem mentalen Lexikon zu schöpfen, sondern durch das implizite Wissen über systematische lautliche Aufeinanderbeziehbarkeit der beiden Sprachen zu konstruieren, was sich auch in hyperkorrekten Formen zeigt. Anhand weiterer Untersuchungen wird zu zeigen sein, ob sich diese Form der kongruenten Lexikalisierung insgesamt als Beschreibungsmodell für die niederdeutschhochdeutsche Sprachmischung vor dem historischen Hintergrund des Schreibsprachenwechsels eignet.

Die in der Salutatio zu beobachtende Sprachmischung ist im Grenzbereich zwischen Codemixing und niederdeutsch-hochdeutscher Mischsprache anzusiedeln, lässt sich aber doch adäquat als Codemixing beschreiben, da sie sich in einem ansonsten niederdeutschen Brief findet. Ob es sich aber noch um Sprachmischung oder schon um eine Mischsprache mit eigenen grammatischen Regeln handelt, lässt sich anhand eines einzelnen Satzes schwer feststellen. Niederdeutsch-hochdeutsche Sprachmischung begegnet aber insgesamt im Stockholmer Material nicht selten, insbesondere in den Rechnungsakten, aber auch in einem anderen Brief an den Ältestenrat der deutschen Gemeinde, nämlich dem bereits mehrfach erwähnten Brief des ebenfalls mit der Stockholmer Gemeinde vertrauten Hannß Bartteram, der vollständig in sprachmischendem Modus abgefasst ist und in einer weiteren Publikation, die sich dem Niederdeutschen in den frühneuzeitlichen Schreiben an den Ältestenrat der Deutschen St. Gertruds Gemeinde widmet, näher behandelt werden soll. An diesem längeren Text kann dann einerseits untersucht werden, ob es sich um eine Mischsprache mit eigenen Regeln handelt, andererseits kann aber auch die hier vorgeschlagene Analyse der Sprachmischung als kongruente Lexikalisierung auf Basis einer geteilten, niederdeutschen grammatischen Struktur, auf etwas breiterer Datenbasis 
überprüft werden. So geben schließlich diese beiden zwar von außen an die Gemeinde gerichteten Briefe - jedoch von Personen, die die Stockholmer Verhältnisse kannten - Aufschluss über den Sprachgebrauch in der Gemeinde selbst und das dortige Nebeneinander von Hoch- und Niederdeutsch. Dies gilt insbesondere, wenn sie eingebettet in weitere Untersuchungen zum Sprachgebrauch in der deutschen Gemeinde betrachtet werden, etwa zur internen Kommunikation in den Rechnungsakten oder zum Sprachgebrauch herausragender Individuen.

\section{Literatur}

Adelung, J. C., 2004. Grammatisch-kritisches Wörterbuch der Hochdeutschen Mundart. Elektronische Volltext- und Faksimile-Edition nach der Ausgabe letzter Hand Leipzig 1793-1801. (Digitale Bibliothek, 40). Berlin: Directmedia.

Ahnlund, N., 1929. "Svenskt och tyskt i Stockholms äldre historia", Historisk tidskrift, 49, S. $1-34$.

Ahnlund, N., 1953. Stockholms historia före Gustav Vasa. Stockholm: Norstedt \& Söner.

Arbusow, L. (Hg.), 1905. Liv-, est- und kurländisches Urkundenbuch. Bd. 2.2: 1501-1505. Riga und Moskau: Kommissions-Verlag von J. Deubner.

Argenter, J. A., 2001. "Code-switching and dialogism: Verbal practices among Catalan Jews in the Middle Ages", Language in Society, 30, S. 377-402.

Auer, P., 1999. “From codeswitching via language mixing to fused lects: Toward a dynamic typology of bilingual speech", International Journal of Bilingualism, 3(4), S. 309-332.

Bein, T., 1998. "Editionsprinzipien für deutsche Texte des späten Mittelalters und der frühen Neuzeit", W. Besch u. a. (Hgg.) Sprachgeschichte: Ein Handbuch zur Geschichte der deutschen Sprache und ihrer Erforschung. Bd. 1. 2., vollst. neu bearb. u. erw. Aufl. (Handbücher zur Sprach- und Kommunikationswissenschaft, 2.1). Berlin und New York: De Gruyter, S. 923-931.

Brattegard, O., 1932. "Über die Organisation und die Urkunden des hansischen Kontors zu Bergen bis 1580", Bergens historiske forening. Skrifter, 38, S. 237-303.

Brattegard, O., 1945. Die mittelniederdeutsche Geschäftssprache des hansischen Kaufmanns zu Bergen. Bd. I:DieSprache der Blütezeit.(Skrifter fra Norges handelshøyskole i rekken språklige avhandlinger, 2). Bergen: A. S. John Griegs boktrykkeri.

Brattegard, O., 1946. Die mittelniederdeutsche Geschäftssprache des hansischen Kaufmanns zu Bergen. Bd. II: Der Ausklang des Niederdeutschen. (Skrifter fra Norges handelshøyskole i rekken språklige avhandlinger, 3). Bergen: A. S. John Griegs boktrykkeri. 
Brattegard, O., 1963. "Niederdeutsch und Norwegisch am hansischen Kontor zu Bergen in Norwegen", Jahrbuch des Vereins für niederdeutsche Sprachforschung, 86, S. 7-16.

Carlsson, G., 1922. "Nicolaus Stecker: Stockholms förste evangeliske kyrkoherde", Kyrkohistorisk Årsskrift, 22, S. 77-93.

Christensen, B., 1993. "Die Verwendung von niederdeutscher und hochdeutscher Sprache in der Deutschen Kanzlei des dänischen Königs im Zeitraum 1540-1549", H. Menke und K. E. Schöndorf (Hgg.) Niederdeutsch in Skandinavien IV: Akten des 4. nordischen Symposions "Niederdeutsch in Skandinavien" in Lübeck-Travemünde 22.-25. August 1991. (Beihefte zur Zeitschrift für deutsche Philologie, 7). Berlin: Erich Schmidt, S. 55-67.

Clyne, M., 2003. Dynamics of Language Contact: English and Immigrant Languages. (Cambridge Approaches to Language Contact). Cambridge u.a.: Cambridge University Press.

Clyne, M. G., 1967. Transference and Triggering: Observations on the Language Assimilation of Postwar German-Speaking Migrants in Australia. The Hague: Martinus Nijhoff.

Dahlbäck, G., 1988. I medeltidens Stockholm. (Monografier utgivna av Stockholms stad, 81). Stockholm: Stockholms medeltidsmuseum.

Edén, N., 1899. Om centralregeringens organisation under den äldre vasatiden (15231594). Dissertation Upsala; Almqvist \& Wiksell: Upsala. Verfügbar unter: http:// runeberg.org/centrorg/ (Zugegriffen: 5.5.2021).

Gabrielsson, A., 1932-1933. "Das Eindringen der hochdeutschen Sprache in die Schulen Niederdeutschlands im 16. und 17. Jahrhundert”, Jahrbuch des Vereins für niederdeutsche Sprachforschung, 58/59, S. 1-79.

Gabrielsson, A., 1983. "Die Verdrängung der mittelniederdeutschen durch die hochdeutsche Schriftsprache", G. Cordes und D. Möhn (Hgg.) Handbuch zur niederdeutschen Sprach- und Literaturwissenschaft. Berlin: Erich Schmidt, S. 119-153.

Glaser, E., M. Prinz und S. Ptashnyk (Hgg.), im Erscheinen. Historisches Codeswitching mit Deutsch: Multilinguale Praktiken in der Sprachgeschichte. (Studia Linguistica Germanica, 140). Berlin und Boston: De Gruyter.

Golz, J., 2007. "Brief", K. Weimar u. a. (Hgg.) Reallexikon der deutschen Literaturwissenschaft. Bd. 1. Neubearbeitung des Reallexikons der deutschen Literaturgeschichte. Berlin und New York: De Gruyter, S. 251-255.

Grimm, J. und W. Grimm, 1984. Deutsches Wörterbuch. Fotomechanischer Nachdruck der Erstausgabe 1854-1971. (33 Bde.). München: dtv.

Heller, M. und C. W. Pfaff, 1996. “Code-switching”, H. Goebl u. a. (Hgg.) Kontaktlinguistik: Ein internationales Handbuch zeitgenössischer Forschung. Bd. 1. (Handbücher zur 
Sprach- und Kommunikationswissenschaft, 12.1). Berlin und New York: De Gruyter, S. 594-6og.

Hellström, G., 1951. Stockholms stads herdaminne från reformationen till tillkomsten av Stockholms stift: Biografisk matrikel. Stockholm.

Kämmerer, C. M., 2006. Codeswitching in Predigten des 15. Jahrhunderts: Mittellatein - Frühneuhochdeutsch. Mittellatein - Altitalienisch/Altspanisch. Zugl. Diss. Zürich 2006. (Studies in Eurolinguistics, 4). Berlin: Logos.

Kantola, M., 1983. "Vruntlike grote to voren: Bemerkungen zum ältesten Niederdeutsch aus Finnland", W. Bahner u. a. (Hgg.) Wissenschaftliche Konferenz "Aspekte und Probleme semasiologischer Sprachbetrachtung in synchronischer und diachronischer Sicht”. Bd. 1. (Linguistische Studien, Reihe A: Arbeitsberichte, 107/I). Berlin: Akademie der Wissenschaften der DDR, S. 16-25.

Katara, P., 1936. "Älteres deutsches Sprachgut und Urkundenmaterial in Finnland", Neuphilologische Mitteilungen, 37(3), S. 161-188.

Katara, [P.], 1950. "Der Ausklang des Mittelniederdeutschen in Finnland", Jahresversammlung des Vereins für niederdeutsche Sprachforschung und des Hansischen Geschichtsvereins, 29.5--1.6.1950, Lemgo. Zitiert nach dem Vortragsresümee von J. Sass: "Jahresversammlung 1950", Korrespondenzblatt des Vereins für niederdeutsche Sprachforschung, 57(2), S. 35-45, hier S. 39-40.

Katara, P. und E. Kuujo, 1958. Deutsche Quittungen in den Rechenschaftsberichten der Vögte Finnlands im 16. Jahrhundert. (Mémoires de la Société Néophilologique de Helsinki, XX,4). Helsinki: Société Néophilologique.

Kjöllerström, S., 1935. Striden kring kalvinismen iSverige under ErikXIV: En kyrkohistorisk studie. (Lunds universitets årsskrift. N. F. Avd. 1). Dissertation Lund; Gleerup: Lund.

Lager, B., 1962. Stockholms befolkning på Johan III:s tid. (Skrifter utgivna av Stadshistoriska institutet). Stockholm: Stadshistoriska institutet.

Lasch, A., 1914. Mittelniederdeutsche Grammatik. (Sammlung kurzer Grammatiken germanischer Dialekte, 9). Halle (Saale): Niemeyer.

Lexer, M., 1992. Mittelhochdeutsches Handwörterbuch: Zugleich als Supplement und alphabetischer Index zum Mittelhochdeutschen Wörterbuche von BeneckeMüller-Zarncke. Nachdruck der Ausg. Leipzig 1872-1878 mit einer Einleitung von Kurt Gärtner. (3 Bde.). Stuttgart: Hirzel.

Lüdeke, Ioan. Ant. Avg., 1791. Dissertatio historica de ecclesia Tevtonica et templo S:tae Gertrvdis Stockholmiensi. Dissertation Upsala; impress. litt. vidvae direct. Ioan. Edman: Upsala.

Lüdeke, J. A. A., 1823. Denkmal der Wieder-Eröffnung der Deutschen Kirche in Stockholm zur öffentlichen Gottes-Verehrung, nach vollendeter Ausbesserung 1821: Eine Predigt mit diplomatisch-historischen Beylagen. Stockholm: gedruckt bei Fr. Bog. Restius. 
Lüdi, G., 1985. "Mehrsprachige Rede in Freiburger Ratsmanualen des 15. Jahrhunderts", Vox Romanica, 44, S. 163-188.

Mähl, S., 2008. geven vnde screven tho deme holme: Variablenlinguistische Untersuchungen zur mittelniederdeutschen Schreibsprache in Stockholm. (Acta Academiae Regiae Gustavi Adolphi, 99). Uppsala: Kungl. Gustav Adolfs Akademien för svensk folkkultur.

Mähl, S., 2012. "Skandinavien", A. Greule, J. Meier, und A. Ziegler (Hgg.) Kanzleisprachenforschung: Ein internationales Handbuch. Berlin und Boston: De Gruyter, S. $623-633$.

Meier, J., 2004. Städtische Kommunikation in der Frühen Neuzeit: Historische Soziopragmatik und Historische Textlinguistik. (Deutsche Sprachgeschichte. Texte und Untersuchungen, 2). Frankfurt a. M. u. a.: Peter Lang.

Murray, R., 1949. Stockholms kyrkostyrelse intill 1630-talets mitt. (Samlingar och studier till Svenska kyrkans historia, 20). Stockholm: Svenska kyrkans diakonistyrelses bokförlag.

Murray, R., 1954. Finska församlingen i Stockholm intill tiden för Finlands avskiljande från Sverige. Stockholm: Svenska kyrkans diakonistyrelses bokförlag.

Muysken, P., 200o. Bilingual Speech: A Typology of Code-Mixing. Cambridge: Cambridge University Press.

Myers-Scotton, C., 1993. Duelling Languages: Grammatical Structures in Codeswitching. Oxford: Clarendon Press.

Nesse, A., 2007. "1750-1850: The dissappearance of German from Bergen, Norway", S. Elspaß u. a. (Hgg.) Germanic Language Histories "from Below" (1700-2000). (Studia Linguistica Germanica, 86). Berlin und New York: De Gruyter, S. 423-435.

Pahta, P., J. Skaffari und L. Wright (Hgg.), 2018. Multilingual Practices in Language History: English and Beyond. (Language Contact and Bilingualism, 15). Boston und Berlin: De Gruyter Mouton.

Peters, R. und N. Nagel, 2018. Fortlaufende Bibliographie zum Schreibsprachenwechsel vom Mittelniederdeutschen, Niederrheinischen und Ripuarischen zum Hochdeutschen und Niederländischen. Münster. Verfügbar unter: https://www.uni-muenster.de/ imperia/md/content/germanistik/lehrende/lehrende/peters_r/3_peters_nagel_sch reibsprachenwechselbibliographie_2004_ff_stand_15022018.pdf (Zugegriffen: 1.5.2021).

Polenz, P. von, 200o. Deutsche Sprachgeschichte vom Spätmittelalter bis zur Gegenwart. Bd. 1: Einführung - Grundbegriffe - 14. bis 16. Jahrhundert. 2., überarb. u. erg. Aufl. Berlin und New York: De Gruyter.

Poplack, S., 1980. "Sometimes I'll start a sentence in Spanish Y TERMINO EN ESPAÑOL: toward a typology of code-switching", Linguistics, 18(7/8), S. 581-618. doi: 10.1515/ ling.1980.18.7-8.581. 
Rothlieb, C. F., 1822. Beskrifning öfver Kongl. Riddarholmskyrkan. Stockholm: tryckt hos J. P. Lindhs enka. Verfügbar unter: https://kulturbilder.wordpress.com/2020/o2/24/ beskrivning-av-riddarholmskyrkan-1822/.

Schendl, H. und L. Wright, 2011a. "Code-switching in early English: Historical background and methodological and theoretical issues", H. Schendl und L. Wright (Hgg.) Code-Switching in Early English. (Topics in English Linguistics, 76). Berlin und Boston: De Gruyter Mouton, S. 15-45.

Schendl, H. und L. Wright (Hgg.), 2011b. Code-Switching in Early English. (Topics in English Linguistics, 76). Berlin und Boston: De Gruyter Mouton.

Schieche, E., 1952. Geschichte der Deutschen St. Gertruds-Gemeinde zu Stockholm. Bd. I: Die Anfänge im 16. Jahrhundert. Münster und Köln: Böhlau-Verlag.

Sidén, P. G., 2008. "Hur tyska var de svenska städerna under högmedeltiden", Svenska historikermötet 2008, 24.-26.4.2008, Lund. Verfügbar unter: https://www.historia .su.se/polopoly_fs/1.27755.1320939842!/SidenTyskaSvenskaStader.pdf (Zugegriffen: 19.4.2021).

Sodmann, T., 1983. "Der Untergang des Mittelniederdeutschen als Schriftsprache", J. Goossens (Hg.) Niederdeutsch: Sprache und Literatur. Eine Einführung. Bd. 1: Sprache. 2. verb. u. um einen bibliogr. Nachtrag erw. Aufl. Neumünster: Wachholtz, S. 116-129.

Sodmann, T., 200o. "Die Verdrängung des Mittelniederdeutschen als Schreib- und Druckersprache Norddeutschlands", W. Besch u. a. (Hgg.) Sprachgeschichte: Ein Handbuch zur Geschichte der deutschen Sprache undihrerErforschung. Bd. 2.2., vollst. neu bearb.u. erw. Aufl. (Handbücher zurSprach- und Kommunikationswissenschaft, 2.2). Berlin und New York: De Gruyter, S. 1505-1512.

Stell, G., 2019. "Code-switching”, J. Darquennes, J. C. Salmons, und W. Vandenbussche (Hgg.) Language Contact: An International Handbook. Bd. 1. (Handbücher zur Sprach- und Kommunikationswissenschaft, 45.1). Berlin und Boston: De Gruyter Mouton, S. 159-171.

Stockholms stadsarkiv (Hg.), 1954. Stockholms stads tänkeböcker från år 1592. Bd. II: 1596-1599. Bearb. v. Daniel Almqvist. Stockholm.

Stockholms stadsarkiv (Hg.), 1957. Stockholms stads tänkeböcker från år 1592. Bd. IV: 1601-1602. Bearb. v. Folke Sleman. Stockholm.

Svalenius, I., 1991. Rikskansliet i Sverige 1560-1592. (Skrifter utgivna av Svenska Riksarkivet, 7). Stockholm: Kommentus.

Upmark, G., 1912. Om Gustaf Vasas hof. Dissertation Upsala; Cederquists: Stockholm.

Weinauge, E., 1942. Die deutsche Bevölkerung im mittelalterlichen Stockholm. (Schriften zur politischen Geschichte und Rassenkunde Schleswig-Holsteins, 5). Leipzig: Hirzel.

Wich-Reif, C. (Hg.), 2016. Historische Sprachkontaktforschung. (Jahrbuch für Germanistische Sprachgeschichte, 7). Berlin und Boston: De Gruyter. 
Winge, V., 1987. "Niederdeutsch-hochdeutscher Sprachwechsel in Dänemark zur Zeit der Reformation und das weitere Schicksal des Niederdeutschen im 17. und 18. Jahrhundert", K. E. Schöndorf und K.-E. Westergard (Hgg.) Niederdeutsch in Skandinavien: Akten des 1. nordischen Symposions "Niederdeutsch in Skandinavien" in Oslo 27.2.-1.3.1985. (Beihefte zur Zeitschrift für deutsche Philologie, 4). Berlin: Erich Schmidt, S. $74-86$. 